\title{
Properties of scattering forms and their relation to associahedra
}

\section{Leonardo de la Cruz, ${ }^{a}$ Alexander Kniss ${ }^{b}$ and Stefan Weinzierl ${ }^{b}$}

${ }^{a}$ Higgs Centre for Theoretical Physics, School of Physics and Astronomy, The University of Edinburgh,

Edinburgh EH9 3JZ, Scotland, U.K.

${ }^{b}$ PRISMA Cluster of Excellence, Institut für Physik,

Johannes Gutenberg-Universität Mainz,

D - 55099 Mainz, Germany

E-mail: leonardo.delacruz@ed.ac.uk, akniss@students.uni-mainz.de, weinzierl@uni-mainz.de

ABSTRACT: We show that the half-integrands in the CHY representation of tree amplitudes give rise to the definition of differential forms - the scattering forms - on the moduli space of a Riemann sphere with $n$ marked points. These differential forms have some remarkable properties. We show that all singularities are on the divisor $\overline{\mathcal{M}}_{0, n} \backslash \mathcal{M}_{0, n}$. Each singularity is logarithmic and the residue factorises into two differential forms of lower points. In order for this to work, we provide a threefold generalisation of the CHY polarisation factor (also known as reduced Pfaffian) towards off-shell momenta, unphysical polarisations and away from the solutions of the scattering equations. We discuss explicitly the cases of bi-adjoint scalar amplitudes, Yang-Mills amplitudes and gravity amplitudes.

Keywords: Scattering Amplitudes, Differential and Algebraic Geometry, Perturbative QCD

ARXIV EPRINT: 1711.07942 


\section{Contents}

1 Introduction 1

2 Review of basic facts 3

2.1 Notation 3

2.2 Amplitudes 5

2.3 The scattering equations 8

2.4 The CHY representation of amplitudes 9

2.5 Multivariate residues 11

3 The moduli space of genus 0 curves with $n$ distinct marked points $\quad 12$

$\begin{array}{ll}3.1 \text { The Deligne-Mumford-Knudsen compactification } & 12\end{array}$

$\begin{array}{lll}3.2 & \text { The dihedral extension } & 14\end{array}$

$\begin{array}{lll}3.3 & \text { The associahedron } & 14\end{array}$

$\begin{array}{lll}3.4 & \text { Coordinates on } \mathcal{M}_{0, z}^{\pi} & 15\end{array}$

4 The scattering forms $\quad 16$

$\begin{array}{ll}4.1 \text { The cyclic scattering form } & 17\end{array}$

4.1.1 Examples 20

$\begin{array}{lll}4.2 & \text { The polarisation scattering form } & 21\end{array}$

$\begin{array}{lll}4.2 .1 & \text { Examples } & 26\end{array}$

$\begin{array}{lll}4.3 & \text { Summary on the factorisation of the residues } & 27\end{array}$

$\begin{array}{lll}5 & \text { Conclusions } & 27\end{array}$

$\begin{array}{lr}\text { A The reduced Pfaffian } & 28\end{array}$

A.1 Definition 28

$\begin{array}{lll}\text { A.2 A counter-example } & 29\end{array}$

B Reduction of the numerators $\quad 29$

$\begin{array}{ll}\text { C Permutation invariance of the polarisation factor } & 30\end{array}$

\section{Introduction}

In this article we bring three things together, which really should be viewed together: (i) the Cachazo-He-Yuan (CHY) representation of tree-level $n$-point scattering amplitudes [1-3], (ii) the moduli space $\mathcal{M}_{0, n}$ of $n$ marked points on a Riemann surface of genus zero and (iii) "positive" geometries / "canonical" forms, as recently discussed by Arkani-Hamed, Bai and Lam [4]. The integrand of the CHY representation for bi-adjoint scalar amplitudes, Yang-Mills amplitudes and gravity amplitudes is constructed from two factors, a cyclic factor (or Parke-Taylor factor) and a polarisation factor (also known as reduced Pfaffian). We show that the cyclic factor and the polarisation factor lead to differential $(n-3)$-forms $\Omega_{\text {scattering }}^{\text {cyclic }}$ and $\Omega_{\text {scattering }}^{\text {pol }}$, respectively, on the compactification $\overline{\mathcal{M}}_{0, n}$ of $\mathcal{M}_{0, n}$, such that the 
only singularities of the differential forms $\Omega_{\text {scattering }}$ are on the divisor $\overline{\mathcal{M}}_{0, n} \backslash \mathcal{M}_{0, n}$. Each singularity is logarithmic and the residue factorises into two differential forms of lower points. These scattering forms figure prominently in the recent work by Mizera [5, 6]. The scattering forms are cocycles and Mizera has shown that the amplitudes are intersection numbers of these cocycles, twisted by a one-form derived from the scattering equations.

We put "positive" geometry into quotes. The reason is the following: the solutions of the scattering equations are in general complex and correspond to points in $\mathcal{M}_{0, n}$. Only for very special external momenta $p$ are the solutions of the scattering equations real [79]. If the solutions are real, we may limit ourselves to the space of real points $\mathcal{M}_{0, n}(\mathbb{R})$. This is a positive space in the sense of Arkani-Hamed, Bai and Lam [4], with boundary $\overline{\mathcal{M}}_{0, n}(\mathbb{R}) \backslash \mathcal{M}_{0, n}(\mathbb{R})$. However, we are interested in the general situation. This forces us to work throughout the paper with the complex numbers $\mathbb{C}$ instead of the real numbers $\mathbb{R}$. For simplicity we write $\mathcal{M}_{0, n}$ instead of $\mathcal{M}_{0, n}(\mathbb{C})$. We find that the notion of "positivity" is not essential, what is essential is the structure of the divisor $\overline{\mathcal{M}}_{0, n} \backslash \mathcal{M}_{0, n}$, which generalises in a straightforward way from the real case $\overline{\mathcal{M}}_{0, n}(\mathbb{R}) \backslash \mathcal{M}_{0, n}(\mathbb{R})$ towards the complex case $\overline{\mathcal{M}}_{0, n}(\mathbb{C}) \backslash \mathcal{M}_{0, n}(\mathbb{C})$.

We also put "canonical" form into quotes. Here, our reason is as follows: the word "canonical" implies, that the differential form is unique for a given geometry. For example, following [4] the differential form for 3 external particles is a 0-form and should for positive geometries be equal to \pm 1 , depending on the orientation. For pseudo-positive geometries the value zero is also allowed. We find this to be the case for $\Omega_{\text {scattering }}^{\text {cyclic }}$ However, we also


amplitude (up to a factor $\pm i$, depending on the orientation). As the geometry of the space always stays the same (we always look at $\mathcal{M}_{0, n}$ ), we are forced to give up the requirement that for $n=3$ the differential 0 -form takes the values $\{-1,0,1\}$ and allow the more general situation that for $n=3$ the differential 0 -form is given by the corresponding three-point amplitude (up to a factor $\pm i$, depending on the orientation).

There is a second - and a slightly more subtle - reason why we put "canonical" into quotes. As mentioned above, for three external gauge bosons the 0 -form $\Omega_{\text {scattering }}^{\text {pol }}$ is given (up to simple proportionality constants) by the three-point amplitude. This is supplemented by an infinite (countable) tower of additional 0 -forms involving auxiliary particles, such that in the factorisation at $n$-points only a finite number of additional 0 -forms occur. The additional 0 -forms vanish for on-shell kinematics, but are non-zero for off-shell kinematics. They are closely related to the construction of BCJ-numerators from an effective Lagrangian. It is known that starting from $n=5$ such an effective Lagrangian is not unique. This implies that for $n \geq 5$ there is more than one possibility to define $\Omega_{\text {scattering }}^{\text {pol }}$.

The CHY representation of Yang-Mills amplitudes and gravity amplitudes involves one or two polarisation factors $E(p, \varepsilon, z)$. The standard definition of the polarisation factor $E(p, \varepsilon, z)$ (for on-shell momenta $p$, transverse polarisations $\varepsilon$ and values $z$ satisfying the scattering equations) is given in terms of a reduced Pfaffian. We will need a threefold generalisation of the polarisation factor: towards off-shell momenta $p$, unphysical polarisations 
$\varepsilon$ and general values $z \in \mathcal{M}_{0, n}$, not restricted to the zero-dimensional sub-variety defined by the scattering equations. We give a definition of the polarisation factor for this general case, which agrees with the reduced Pfaffian for on-shell momenta, transverse polarisations and on the sub-variety defined by the scattering equations.

Let us mention that shortly after our paper appeared on the arXive, ref. [10] appeared on the arXive, where scattering forms on the kinematic space of Mandelstam variables are studied. Ref. [10] defines a kinematic associahedron, where positivity conditions for all planar Lorentz invariants $s_{i_{1}, \ldots, i_{k}}$ and constraints $-s_{i j}=c_{i j}>0$ for non-adjacent Lorentz invariants with $1 \leq i<j \leq(n-1)$ are imposed. With these constraints, the scattering forms on the kinematic space are related by a push-forward to the scattering forms discussed in our paper. In this paper we do not impose the positivity conditions nor the constraints $-s_{i j}=c_{i j}>0$.

This paper is organised as follows: in section 2 we introduce our notation and review a few basic concepts. The moduli space $\mathcal{M}_{0, n}$ of $n$ marked points on a Riemann surface of genus zero and its compactification $\overline{\mathcal{M}}_{0, n}$ plays a prominent role in this paper and we review the definition and essential properties in section 3. Section 4 contains the main part of this paper. We study the scattering forms and exhibit some remarkable properties. Our conclusions are contained in section 5. In the appendix we collected useful information on the standard definition of the reduced Pfaffian and technical details on proofs.

\section{Review of basic facts}

\section{$2.1 \quad$ Notation}

Let us consider a scattering process with $n$ massless particles in $D$ space-time dimensions within the Born approximation. We denote the momenta of the external particles by $p=$ $\left\{p_{1}, \ldots, p_{n}\right\}$. By convention we take all momenta to be outgoing, momentum conservation reads therefore

$$
\sum_{i=1}^{n} p_{i}=0 .
$$

If all external particles are gauge bosons, we denote by $\varepsilon=\left(\varepsilon_{1}, \ldots, \varepsilon_{n}\right)$ the polarisation vectors.

Let $I$ be a subset of $\{1,2, \ldots, n\}$. We set

$$
p_{I}=\sum_{i \in I} p_{i}
$$

and

$$
s_{I}=p_{I}^{2}
$$

Let $\sigma=\left(\sigma_{1}, \ldots, \sigma_{n}\right)$ be a permutation of $(1, \ldots, n)$. A cyclic order is defined as a permutation modulo cyclic permutations $\left(\sigma_{1}, \sigma_{2}, \ldots, \sigma_{n}\right) \rightarrow\left(\sigma_{2}, \ldots, \sigma_{n}, \sigma_{1}\right)$. We may represent a cyclic order by an $n$-gon, where the edges of the $n$-gon are indexed clockwise by $\sigma_{1}, \sigma_{2}$, $\ldots, \sigma_{n}$. A dihedral structure is defined as a permutation modulo cyclic permutations and reflection $\left(\sigma_{1}, \sigma_{2}, \ldots, \sigma_{n}\right) \rightarrow\left(\sigma_{n}, \ldots, \sigma_{2}, \sigma_{1}\right)$. We may represent a dihedral structure by an 
$n$-gon, where the edges of the $n$-gon are indexed either clockwise or anti-clockwise by $\sigma_{1}$, $\sigma_{2}, \ldots, \sigma_{n}$. This is illustrated in the left picture of figure 2 .

We will need a few definitions related to graphs. In the following we consider tree graphs with $n$ external legs. For a graph $G$ we denote by $E(G)$ the set of the internal edges and by $s_{e}$ the Lorentz invariant corresponding to the internal edge $e$.

We denote by $\mathcal{T}_{n}(\sigma)$ the set of all cyclic ordered tree diagrams with trivalent vertices and external cyclic order $\sigma$. The number of graphs in the set $\mathcal{T}_{n}(\sigma)$ is given by

$$
\left|\mathcal{T}_{n}(\sigma)\right|=\frac{(2 n-4) !}{(n-2) !(n-1) !} .
$$

Two diagrams with different external orders are considered to be equivalent, if we can transform one diagram into the other by a sequence of flips. Under a flip operation one exchanges at a vertex two branches. We denote by $\mathrm{CO}(G)$ the set of cyclic orders obtained from the graph $G$ by flipping in all possible ways the branches at the vertices. The number of cyclic orders in the set $\mathrm{CO}(G)$ is given by

$$
|\mathrm{CO}(G)|=2^{n-2}
$$

We denote by $\mathcal{T}_{n}(\sigma) \cap \mathcal{T}_{n}(\tilde{\sigma})$ the set of graphs compatible with the external orders $\sigma$ and $\tilde{\sigma}$ and by $n_{\text {flip }}(\sigma, \tilde{\sigma})$ the number of flips needed to transform any graph from $\mathcal{T}_{n}(\sigma) \cap \mathcal{T}_{n}(\tilde{\sigma})$ with the external order $\sigma$ into a graph with the external order $\tilde{\sigma}$. The number $n_{\text {flip }}(\sigma, \tilde{\sigma})$ will be the same for all graphs from $\mathcal{T}_{n}(\sigma) \cap \mathcal{T}_{n}(\tilde{\sigma})$.

We denote by $\mathcal{U}_{n}$ the set of all unordered tree graphs with trivalent vertices. The number of graphs in this set is given by

$$
\left|\mathcal{U}_{n}\right|=(2 n-5) ! !=2^{2-n} \frac{(2 n-4) !}{(n-2) !} .
$$

Let $G^{\text {unordered }} \in \mathcal{U}_{n}$ be an unordered tree graph. We may draw this graph as a cyclic ordered graph in $2^{n-2}$ different ways. Let $G$ be one possibility. We denote by $\operatorname{co}(G)$ the cyclic order of $G$. If $G^{\prime}$ is another possibility of drawing $G^{\text {unordered }}$ as a cyclic ordered graph, the relative sign between the two graphs is given by

$$
(-1)^{n_{\text {flip }}\left(\operatorname{co}(G), \operatorname{co}\left(G^{\prime}\right)\right)} \text {. }
$$

The following formula is useful to exchange summation orders:

$$
\sum_{\sigma \in S_{n} / \mathbb{Z}_{n}} \sum_{G \in \mathcal{T}_{n}(\sigma)}=\sum_{G \in \mathcal{U}_{n}} \sum_{\sigma \in \mathrm{CO}(G)}
$$

This formula says that it is equivalent either to sum first over all cyclic orders and then within a given cyclic order over all graphs contributing to it, or to sum first over all unordered graphs and then over all cyclic orders compatible with this graph. In practice the summands will depend on cyclic ordered graphs. For unordered graphs we will have to pick a cyclic ordered representative and take relative signs between equivalent representatives into account. 
Let us also introduce a notation to specify tree graphs with a fixed cyclic order and trivalent vertices. We follow ref. [11]. Let us assume that the cyclic order is $(1,2, \ldots, n)$. If we single out one specific external leg (usually we take the last leg $n$ ), we speak of a rooted tree, the root being given by the external leg which we singled out. We may specify a rooted tree by brackets involving the remaining legs, for example

$$
[[1,2], 3]
$$

denotes the rooted tree

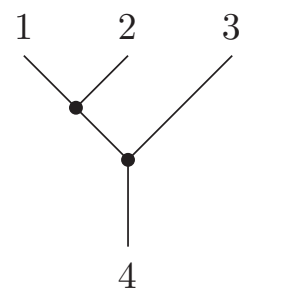

In addition we consider non-rooted trees. We define a concatenation operation for two rooted trees: let $T_{1}$ and $T_{2}$ be two rooted trees with roots $r_{1}$ and $r_{2}$. Then we denote by $\left(T_{1}, T_{2}\right)$ the non-rooted tree obtained from $T_{1}$ and $T_{2}$ by joining the two roots by an edge. As an example we have:

$$
(\overbrace{r_{1}}^{1}, \gamma_{r_{2}}^{2})={ }_{1}^{2} \gamma_{4}^{3}
$$

The concatenation operation is symmetric:

$$
\left(T_{1}, T_{2}\right)=\left(T_{2}, T_{1}\right) .
$$

If $T_{1}, T_{2}$ and $T_{3}$ are rooted trees, we have the obvious relations

$$
\left(\left[T_{1}, T_{2}\right], T_{3}\right)=\left(\left[T_{2}, T_{3}\right], T_{1}\right)=\left(\left[T_{3}, T_{1}\right], T_{2}\right) .
$$

Every rooted tree can be viewed as a non-rooted tree by simply forgetting that one external leg has been marked as a root.

\section{$2.2 \quad$ Amplitudes}

In this paper we consider the bi-adjoint scalar amplitudes $m_{n}(\sigma, \tilde{\sigma}, p)$, the Yang-Mills amplitudes $A_{n}(\sigma, p, \varepsilon)$ and the graviton amplitudes $M_{n}(p, \varepsilon, \tilde{\varepsilon})$. The bi-adjoint scalar amplitude $m_{n}(\sigma, \tilde{\sigma}, p)$ depends on two cyclic orders $\sigma$ and $\tilde{\sigma}$ and arises in the double colour decomposition of the full bi-adjoint tree amplitude:

$$
m_{n}(p)=\lambda^{n-2} \sum_{\sigma \in S_{n} / Z_{n}} \sum_{\tilde{\sigma} \in S_{n} / Z_{n}} 2 \operatorname{Tr}\left(T^{a_{\sigma(1)}} \ldots T^{a_{\sigma(n)}}\right) 2 \operatorname{Tr}\left(\tilde{T}^{b_{\tilde{\sigma}(1)}} \ldots \tilde{T}^{b_{\tilde{\sigma}(n)}}\right) m_{n}(\sigma, \tilde{\sigma}, p)
$$


The double-ordered amplitude $m_{n}(\sigma, \tilde{\sigma}, p)$ is rather simple and explicitly given by

$$
m_{n}(\sigma, \tilde{\sigma}, p)=i(-1)^{n-3+n_{\text {flip }}(\sigma, \tilde{\sigma})} \sum_{G \in \mathcal{T}_{n}(\sigma) \cap \mathcal{T}_{n}(\tilde{\sigma})} \prod_{e \in E(G)} \frac{1}{s_{e}} .
$$

The partial Yang-Mills amplitude $A_{n}(\sigma, p, \varepsilon)$ appears in the (single) colour decomposition of the full Yang-Mills tree amplitude:

$$
\mathcal{A}_{n}(p, \varepsilon)=g^{n-2} \sum_{\sigma \in S_{n} / Z_{n}} 2 \operatorname{Tr}\left(T^{a_{\sigma(1)}} \ldots T^{a_{\sigma(n)}}\right) \quad A_{n}(\sigma, p, \varepsilon)
$$

It is well known that we may eliminate the four-gluon vertex in Yang-Mills theory by introducing an auxiliary tensor particle [12-14] with propagator

$$
[\mu \nu]=[\rho \sigma]=-\frac{i}{2}\left(g_{\mu \rho} g_{\nu \sigma}-g_{\mu \sigma} g_{\nu \rho}\right),
$$

and vertex

$$
\prod_{3,[\rho \sigma]}^{1, \mu e_{0}} \sigma^{2, \nu}=\frac{i}{\sqrt{2}}\left(g^{\mu \rho} g^{\nu \sigma}-g^{\mu \sigma} g^{\nu \rho}\right) \text {. }
$$

This allows us to compute $A_{n}(\sigma, p, \varepsilon)$ from tree diagrams with trivalent vertices only. Thus we may write

$$
A_{n}=i(-1)^{n-3} \sum_{G \in \mathcal{T}_{n}(\sigma)} N^{\text {Feynman }}(G) \prod_{e \in E(G)} \frac{1}{s_{e}},
$$

where the numerators $N^{\text {Feynman }}(G)$ are given by Feynman rules. Note that the numerators are not BCJ-numerators. Although they do satisfy the anti-symmetry relations, they do in general not satisfy Jacobi relations. It is however possible [15-17] to express $A_{n}$ in a form similar to eq. (2.19)

$$
A_{n}=i(-1)^{n-3} \sum_{G \in \mathcal{T}_{n}(\sigma)} N^{\mathrm{BCJ}}(G) \prod_{e \in E(G)} \frac{1}{s_{e}}
$$

with numerators $N^{\mathrm{BCJ}}(G)$ satisfying anti-symmetry relations and Jacobi relations. It is further possible to obtain the BCJ-numerators from an effective Lagrangian $[11,17]$

$$
\mathcal{L}_{\mathrm{YM}}+\mathcal{L}_{\mathrm{GF}}=\frac{1}{2 g^{2}} \sum_{n=2}^{\infty} \mathcal{L}^{(n)}
$$

where $\mathcal{L}^{(n)}$ contains $n$ fields. The terms $\mathcal{L}^{(2)}, \mathcal{L}^{(3)}$ and $\mathcal{L}^{(4)}$ agree with the standard terms. Let us introduce the Lie-algebra valued field

$$
\mathbf{A}_{\mu}=\frac{g}{i} T^{a} A_{\mu}^{a}
$$


Then the terms $\mathcal{L}^{(2)}, \mathcal{L}^{(3)}$ and $\mathcal{L}^{(4)}$ are given by

$$
\begin{aligned}
& \mathcal{L}^{(2)}=-2 \operatorname{Tr} \mathbf{A}_{\mu} \square \mathbf{A}^{\mu}, \\
& \mathcal{L}^{(3)}=4 \operatorname{Tr}\left(\partial_{\mu} \mathbf{A}_{\nu}\right)\left[\mathbf{A}^{\mu}, \mathbf{A}^{\nu}\right] \\
& \mathcal{L}^{(4)}=-g^{\mu_{1} \mu_{3}} g^{\mu_{2} \mu_{4}} g_{\nu_{1} \nu_{2}} \frac{\partial_{12}^{\nu_{1}} \partial_{34}^{\nu_{2}}}{\square_{12}} \operatorname{Tr}\left[\mathbf{A}_{\mu_{1}}, \mathbf{A}_{\mu_{2}}\right]\left[\mathbf{A}_{\mu_{3}}, \mathbf{A}_{\mu_{4}}\right] .
\end{aligned}
$$

The subscripts on the derivatives indicate on which fields they act. For $\mathcal{L}^{(4)}$ we have introduced a factor $1 / \square_{12}$, which assigns an intermediate propagator to the four-gluon vertex. Note that this factor cancels against the factor $\left(-g_{\nu_{1} \nu_{2}} \partial_{12}^{\nu_{1}} \partial_{34}^{\nu_{2}}\right)=\square_{12}$. Leaving the two factors uncancelled keeps the information on the assignment of terms to diagrams with three-valent vertices only. This corresponds exactly to the introduction of an auxiliary particle through eq. (2.17) and eq. (2.18).

The terms $\mathcal{L}^{(n)}$ for $n \geq 5$ are equivalent to zero. They ensure that BCJ-numerators are obtained from the Feynman rules. To give an example we may take for $\mathcal{L}^{(5)}$

$$
\begin{aligned}
\mathcal{L}^{(5)}= & 4 g^{\mu_{1} \mu_{3}} g^{\mu_{2} \mu_{4}} \frac{\partial_{1}^{\mu_{5}}}{\square_{123}}\left(\operatorname{Tr}\left[\left[\left[\mathbf{A}_{\mu_{1}}, \mathbf{A}_{\mu_{2}}\right], \mathbf{A}_{\mu_{3}}\right], \mathbf{A}_{\mu_{4}}\right] \mathbf{A}_{\mu_{5}}\right. \\
& \left.+\operatorname{Tr}\left[\left[\mathbf{A}_{\mu_{3}}, \mathbf{A}_{\mu_{4}}\right],\left[\mathbf{A}_{\mu_{1}}, \mathbf{A}_{\mu_{2}}\right]\right] \mathbf{A}_{\mu_{5}}+\operatorname{Tr}\left[\left[\mathbf{A}_{\mu_{4}},\left[\mathbf{A}_{\mu_{1}}, \mathbf{A}_{\mu_{2}}\right]\right], \mathbf{A}_{\mu_{3}}\right] \mathbf{A}_{\mu_{5}}\right) .
\end{aligned}
$$

The term $\mathcal{L}^{(5)}$ is equal to zero due to the Jacobi identity involving the expressions $\left[\mathbf{A}_{\mu_{1}}, \mathbf{A}_{\mu_{2}}\right], \mathbf{A}_{\mu_{3}}$ and $\mathbf{A}_{\mu_{4}}$. However, the term $\mathcal{L}^{(5)}$ generates a five-valent vertex. This five-valent vertex gives a non-vanishing contribution to individual numerators. In a partial amplitude the sum of all terms related to the five-valent vertex adds up to zero. We note that the terms $\mathcal{L}^{(n)}$ for $n \geq 5$ are not unique. For example, we may replace in eq. (2.24)

$$
4 g^{\mu_{1} \mu_{3}} g^{\mu_{2} \mu_{4}} \partial_{1}^{\mu_{5}} \rightarrow 4 g^{\mu_{1} \mu_{3}} g^{\mu_{2} \mu_{4}} \partial_{1}^{\mu_{5}}-4 a\left(g^{\mu_{1} \mu_{3}} g^{\mu_{2} \mu_{4}} \partial_{1}^{\mu_{5}}-g^{\mu_{1} \mu_{3}} g^{\mu_{4} \mu_{5}} \partial_{4}^{\mu_{2}}\right),
$$

where $a$ is a free parameter.

From the effective Lagrangian in eq. (2.21) we obtain BCJ-numerators, which are polynomials in $p$ and $\varepsilon$. The BCJ-numerators $N^{\mathrm{BCJ}}(G)$ inherit their graph structure from the underlying graph $G$. Let $I$ be a subset of $\{1,2, \ldots, n\}$. We say that $N^{\mathrm{BCJ}}(G)$ factorises in the channel $I$, if there is an edge $e \in G$, such that $s_{I}=s_{e}$, otherwise we say that $N^{\mathrm{BCJ}}(G)$ does not factorise in the channel $I$. In other words, the factorisation channels of a graph $G$ correspond exactly to the internal edges (or propagators) of the graph.

Combining the anti-symmetry of the vertices and the Jacobi identity one has
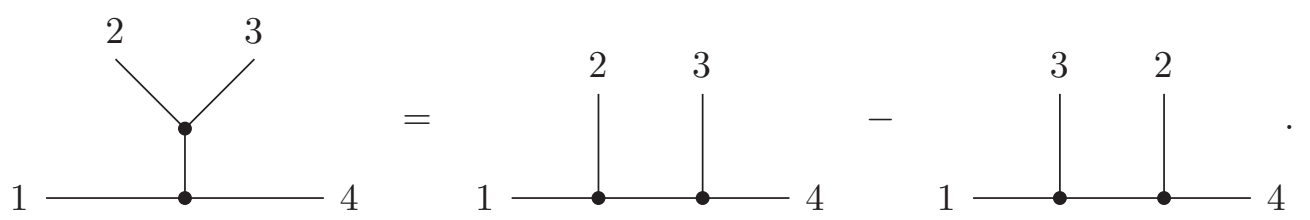

Eq. (2.26) is called a STU-relation. With the help of eq. (2.26) we may reduce any tree graph with $n$ external legs and containing only trivalent vertices to a multi-peripheral form with respect to 1 and $n$. We say that a graph is multi-peripheral (or in comb form) with respect to 1 and $n$, if all other external legs connect directly to the line from 1 to $n$, i.e. 
there are no non-trivial sub-trees attached to this line. A graph in multi-peripheral form can be drawn as

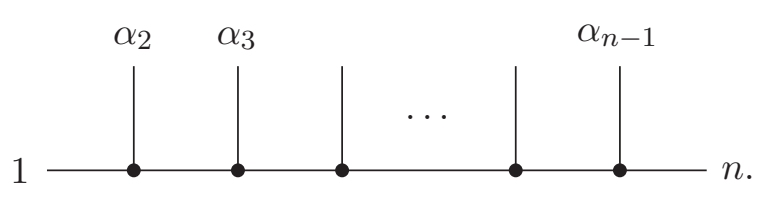

Let us set $\kappa=(1, \alpha, n)$. We denote the BCJ-numerator of a multi-peripheral graph by

$$
N_{\text {comb }}^{\mathrm{BCJ}}(\kappa) \text {. }
$$

There are $(n-2)$ ! multi-peripheral BCJ-numerators, indexed by a permutation $\left(\alpha_{2}, \ldots, \alpha_{n-1}\right)$ of $(2, \ldots, n-1)$.

After this excursion towards BCJ-numerators let us turn back to scattering amplitudes. The graviton amplitude $M_{n}(p, \varepsilon, \tilde{\varepsilon})$ is obtained by expanding the Einstein-Hilbert action

$$
\mathcal{L}_{\mathrm{EH}}=-\frac{2}{\kappa^{2}} \sqrt{-g} R
$$

around the flat Minkowski metric

$$
g_{\mu \nu}=\eta_{\mu \nu}+\kappa h_{\mu \nu}
$$

$h_{\mu \nu}$ is the graviton field. The polarisation of a graviton is described by a product of two spin-1 polarisation vectors:

$$
\text { either } \varepsilon_{\mu}^{+} \tilde{\varepsilon}_{\nu}^{+} \text {or } \varepsilon_{\mu}^{-} \tilde{\varepsilon}_{\nu}^{-} \text {. }
$$

For $n$ external gravitons we collect the first polarisation vector in $\varepsilon$, and the second polarisation vector in $\tilde{\varepsilon}$.

\subsection{The scattering equations}

Let us consider $z=\left(z_{1}, z_{2}, \ldots, z_{n}\right) \in\left(\mathbb{C P}^{1}\right)^{n}$ with $z_{i} \neq z_{j}$. One defines for $1 \leq i \leq n$

$$
f_{i}(z, p)=\sum_{j=1, j \neq i}^{n} \frac{2 p_{i} \cdot p_{j}}{z_{i}-z_{j}} .
$$

The scattering equations are given by $[1-3]$

$$
f_{i}(z, p)=0, \quad i \in\{1, \ldots, n\} .
$$

For fixed momenta $p$ a solution of the scattering equations is a point $z \in\left(\mathbb{C P}^{1}\right)^{n}$, such that the scattering equations in eq. (2.33) are satisfied.

The scattering equations are invariant under the projective special linear group $\operatorname{PSL}(2, \mathbb{C})=\operatorname{SL}(2, \mathbb{C}) / \mathbb{Z}_{2}$. Here, $\mathbb{Z}_{2}$ is given by $\{\mathbf{1},-\mathbf{1}\}$, with $\mathbf{1}$ denoting the unit matrix. The matrix

$$
g=\left(\begin{array}{ll}
a & b \\
c & d
\end{array}\right) \in \operatorname{PSL}(2, \mathbb{C})
$$


acts on all $z_{j}$ by

$$
g \cdot z_{i}=\frac{a z_{i}+b}{c z_{i}+d}, \quad i \in\{1, \ldots, n\} .
$$

We write $g \cdot\left(z_{1}, \ldots, z_{n}\right)=\left(g \cdot z_{1}, \ldots, g \cdot z_{n}\right)$. If $\left(z_{1}, z_{2}, \ldots, z_{n}\right)$ is a solution of eq. (2.33), then also $\left(z_{1}^{\prime}, z_{2}^{\prime}, \ldots, z_{n}^{\prime}\right)=g \cdot\left(z_{1}, z_{2}, \ldots, z_{n}\right)$ is a solution. Two solutions which are related by a $\operatorname{PSL}(2, \mathbb{C})$-transformation are called equivalent solutions. From Bézout's theorem [18] it follows that there are $(n-3)$ ! inequivalent solutions of the scattering equations not related by a $\operatorname{PSL}(2, \mathbb{C})$-transformation.

Following [5] one defines a one-form $\eta$ by

$$
\eta=\sum_{i=1}^{n} f_{i}(z, p) d z_{i}
$$

The one-form $\eta$ defines the twist, such that amplitudes are expressed as twisted intersection numbers of two cocycles. The two cocycles are differential $(n-3)$-forms, and their properties are the subject of this paper.

\subsection{The CHY representation of amplitudes}

There are a large variety of theories, in which the Born amplitudes have a CHY representation, i.e. the Born amplitudes can be written in the form

$$
A_{n}=i \oint_{\mathcal{C}} d \Omega_{\mathrm{CHY}} F(z) .
$$

The measure $d \Omega_{\mathrm{CHY}}$ is defined by

$$
d \Omega_{\mathrm{CHY}}=\frac{1}{(2 \pi i)^{n-3}} \frac{d^{n} z}{d \omega} \prod^{\prime} \frac{1}{f_{a}(z, p)},
$$

where

$$
\prod^{\prime} \frac{1}{f_{a}(z, p)}=(-1)^{i+j+k}\left(z_{i}-z_{j}\right)\left(z_{j}-z_{k}\right)\left(z_{k}-z_{i}\right) \prod_{a \neq i, j, k} \frac{1}{f_{a}(z, p)}
$$

and

$$
d \omega=(-1)^{p+q+r} \frac{d z_{p} d z_{q} d z_{r}}{\left(z_{p}-z_{q}\right)\left(z_{q}-z_{r}\right)\left(z_{r}-z_{q}\right)} .
$$

The primed product of $1 / f_{a}$ is independent of the choice of $i, j, k$ and takes into account that only $(n-3)$ of the scattering equations are independent. The quantity $d \omega$ is independent of the choice of $p, q, r$ and corresponds to the invariant measure on $\operatorname{PSL}(2, \mathbb{C})$. The integration contour $\mathcal{C}$ encircles the inequivalent solutions of the scattering equations. Under PSL $(2, \mathbb{C})$-transformations the integrand $F(z)$ transforms as

$$
F(g \cdot z)=\left(\prod_{j=1}^{n}\left(c z_{j}+d\right)^{4}\right) F(z)
$$


It is often the case that $F(z)$ factors

$$
F(z)=F_{1}(z) F_{2}(z)
$$

where each factor transforms as

$$
F_{i}(g \cdot z)=\left(\prod_{j=1}^{n}\left(c z_{j}+d\right)^{2}\right) F_{i}(z), \quad i \in\{1,2\} .
$$

We call the factors $F_{1}(z)$ and $F_{2}(z)$ half-integrands. In this paper we consider the standard examples of amplitudes in a bi-adjoint scalar theory, Yang-Mills theory and gravity. For these amplitudes the $\mathrm{CHY}$ integrand is constructed from two building blocks, a cyclic factor $C(\sigma, z)$ defined by

$$
C(\sigma, z)=\frac{1}{\left(z_{\sigma_{1}}-z_{\sigma_{2}}\right)\left(z_{\sigma_{2}}-z_{\sigma_{3}}\right) \ldots\left(z_{\sigma_{n}}-z_{\sigma_{1}}\right)}
$$

and a polarisation factor $E(p, \varepsilon, z)$. The original definition of the polarisation factor in terms of a reduced Pfaffian is given in appendix A. The CHY integrands are then given by

$$
\begin{aligned}
F_{\text {bi-adjoint }} & =C(\sigma, z) C(\tilde{\sigma}, z), \\
F_{\mathrm{YM}} & =C(\sigma, z) E(p, \varepsilon, z), \\
F_{\text {gravity }} & =E(p, \varepsilon, z) E(p, \tilde{\varepsilon}, z) .
\end{aligned}
$$

Each half-integrand defines a $(n-3)$-form

$$
\begin{aligned}
\Omega_{\text {scattering }}^{\text {cyclic }}(\sigma, z) & =C(\sigma, z) \frac{d^{n} z}{d \omega}, \\
\Omega_{\text {scattering }}^{\text {pol }}(p, \varepsilon, z) & =E(p, \varepsilon, z) \frac{d^{n} z}{d \omega} .
\end{aligned}
$$

Mizera [5] has shown recently that the amplitudes are given as twisted intersection numbers of these forms, twisted by the one-form $\eta$, i.e.

$$
\begin{aligned}
m_{n}(\sigma, \tilde{\sigma}, p) & =i\left(\Omega_{\text {scattering }}^{\text {cyclic }}(\sigma, z), \Omega_{\text {scattering }}^{\text {cyclic }}(\tilde{\sigma}, z)\right)_{\eta}, \\
A_{n}(\sigma, p, \varepsilon) & =i\left(\Omega_{\text {scattering }}^{\text {cyclic }}(\sigma, z), \Omega_{\text {scattering }}^{\text {pol }}(p, \varepsilon, z)\right)_{\eta} \\
M_{n}(p, \varepsilon, \tilde{\varepsilon}) & =i\left(\Omega_{\text {scattering }}^{\text {pol }}(p, \varepsilon, z), \Omega_{\text {scattering }}^{\text {pol }}(p, \tilde{\varepsilon}, z)\right)_{\eta},
\end{aligned}
$$

where $(A, B)_{\eta}$ denotes the intersection number of two cocycles twisted by $\eta$. Eq. (2.47) depends only on the values of the scattering forms on the sub-variety defined by the scattering equations. However, the scattering forms $\Omega_{\text {scattering }}^{\text {cycli }}$ and $\Omega_{\text {scattering }}^{\text {pol }}$ themselves do not know about the scattering equations. The scattering equations enter only through the twist $\eta$. It turns out that the cyclic scattering form $\Omega_{\text {scattering }}^{\text {cyclic }}$ has in addition nice mathematical properties away from the zero-dimensional sub-variety defined by the scattering equations. In this paper we study these properties. We also show that with a suitable re-definition of the polarisation factor $E(p, \varepsilon, z)$ the same properties hold for the scattering form $\Omega_{\mathrm{scattering}}^{\mathrm{pol}}$. 


\subsection{Multivariate residues}

In this paragraph we review multivariate residues of differential forms. We follow ref. [19]. Let $X$ be a $n$-dimensional variety and $Y$ a co-dimension one sub-variety. Let us choose a coordinate system such that $Y$ is given locally by $z_{1}=0$. Assume that $\Omega$ has a pole of order $k$ on $Y$. Then $\Omega$ may be written as

$$
\Omega=\frac{d z_{1}}{z_{1}^{k}} \wedge \psi+\theta
$$

where the $(n-1)$-form $\psi$ is regular and non-vanishing on $Y$, and the $n$-form $\theta$ has at most a pole of order $(k-1)$ on $Y$. We may reduce poles of order $k>1$ to poles of order 1 and exact forms due to the identity

$$
\frac{d z_{1}}{z_{1}^{k}} \wedge \psi+\theta=d\left(-\frac{\psi}{(k-1) z_{1}^{k-1}}\right)+\frac{d \psi}{(k-1) z_{1}^{k-1}}+\theta
$$

Thus every form $\Omega$ is equivalent (up to an exact form) to a form $\Omega_{1}$ with at most a single pole on $Y$. For

$$
\Omega_{1}=\frac{d z_{1}}{z_{1}} \wedge \psi_{1}+\theta_{1}
$$

we set

$$
\operatorname{Res}_{Y}\left(\Omega_{1}\right)=\left.\psi_{1}\right|_{Y},
$$

and if $\Omega_{1}$ is equivalent to $\Omega$ up to an exact form

$$
\operatorname{Res}_{Y}(\Omega)=\operatorname{Res}_{Y}\left(\Omega_{1}\right)
$$

Multivariate residues are defined as follows: suppose we have two co-dimension one subvarieties $Y_{1}$ and $Y_{2}$ defined by $z_{1}=0$ and $z_{2}=0$, respectively. Again we may reduce higher poles to simple poles modulo exact forms. Let us therefore consider

$$
\Omega=\frac{d z_{1}}{z_{1}} \wedge \frac{d z_{2}}{z_{2}} \wedge \psi_{12}+\frac{d z_{1}}{z_{1}} \wedge \psi_{1}+\frac{d z_{2}}{z_{2}} \wedge \psi_{2}+\theta
$$

where $\psi_{12}$ is regular on $Y_{1} \cap Y_{2}, \psi_{j}$ is regular on $Y_{j}$ and $\theta$ is regular on $Y_{1} \cup Y_{2}$. One sets

$$
\operatorname{Res}_{Y_{1}, Y_{2}}(\Omega)=\left.\psi_{12}\right|_{Y_{1} \cap Y_{2}}
$$

Note that the residue is anti-symmetric with respect to the order of the hypersurfaces:

$$
\operatorname{Res}_{Y_{2}, Y_{1}}(\Omega)=-\operatorname{Res}_{Y_{1}, Y_{2}}(\Omega) .
$$

Multivariate residues for several co-dimension one sub-varieties $Y_{1}, \ldots, Y_{m}$ are defined analogously. 



Figure 1. The moduli space $\mathcal{M}_{0,5}(\mathbb{R})$ (left). The region $X$ is bounded by $z_{2}=0, z_{3}=1$ and $z_{2}=z_{3}$. The right figure shows $\overline{\mathcal{M}}_{0,5}(\mathbb{R})$, obtained from $\mathcal{M}_{0,5}(\mathbb{R})$ by blowing up the points $\left(z_{2}, z_{3}\right)=(0,0),\left(z_{2}, z_{3}\right)=(1,1)$ and $\left(z_{2}, z_{3}\right)=(\infty, \infty)$.

\section{The moduli space of genus 0 curves with $n$ distinct marked points}

Let us consider a Riemann sphere (i.e. an algebraic curve of genus zero) with $n$ distinct marked points. The moduli space of genus 0 curves with $n$ distinct marked points is denoted by

$$
\mathcal{M}_{0, n}=\left\{z \in\left(\mathbb{C P}^{1}\right)^{n}: z_{i} \neq z_{j}\right\} / \operatorname{PSL}(2, \mathbb{C}) .
$$

$\mathcal{M}_{0, n}$ is an affine algebraic variety of dimension $(n-3)$. We may use the freedom of $\operatorname{PSL}(2, \mathbb{C})$-transformations to fix three points. The standard choice will be $z_{1}=0, z_{n-1}=1$ and $z_{n}=\infty$. Thus

$$
\mathcal{M}_{0, n}=\left\{\left(z_{2}, \ldots, z_{n-2}\right) \in \mathbb{C}^{n-3}: z_{i} \neq z_{j}, z_{i} \neq 0, z_{i} \neq 1\right\} .
$$

We denote the set of real points by $\mathcal{M}_{0, n}(\mathbb{R})$ :

$$
\mathcal{M}_{0, n}(\mathbb{R})=\left\{\left(z_{2}, \ldots, z_{n-2}\right) \in \mathbb{R}^{n-3}: z_{i} \neq z_{j}, z_{i} \neq 0, z_{i} \neq 1\right\} .
$$

In figure 1 we sketch the moduli space $\mathcal{M}_{0,5}(\mathbb{R})$. In this example the region $X$ is bounded by $z_{2}=0, z_{3}=1$ and $z_{2}=z_{3}$. In general there will be points, where the boundaries do not cross normally. For the region $X$ in the example above this occurs for $\left(z_{2}, z_{3}\right)=(0,0)$ and $\left(z_{2}, z_{3}\right)=(1,1)$. We denote by $\overline{\mathcal{M}}_{0, n}$ the blow-up of $\mathcal{M}_{0, n}$ in all those points, such that in $\overline{\mathcal{M}}_{0, n}$ all boundaries cross normally. In this way the region $X$ of our example transforms from a triangle in $\mathcal{M}_{0,5}(\mathbb{R})$ into a pentagon in $\overline{\mathcal{M}}_{0,5}(\mathbb{R})$.

\subsection{The Deligne-Mumford-Knudsen compactification}

Let us now review a systematic way to construct $\overline{\mathcal{M}}_{0, n}$. There is a smooth compactification

$$
\mathcal{M}_{0, n} \subset \overline{\mathcal{M}}_{0, n}
$$





Figure 2. A hexagon, where the edges are labelled by the cyclic ordered variables $\left(z_{1}, z_{2}, \ldots, z_{6}\right)$ (left picture). The middle picture shows the chord $(2,5)$. Right picture: a chord divides the hexagon into two lower $n$-gons, in this case two quadrangles.

known as the Deligne-Mumford-Knudsen compactification [20-23], such that $\overline{\mathcal{M}}_{0, n} \backslash \mathcal{M}_{0, n}$ is a smooth normal crossing divisor. In order to describe $\overline{\mathcal{M}}_{0, n}$ we follow ref. [24]. The construction proceeds through intermediate spaces $\mathcal{M}_{0, n}^{\pi}$, labelled by a dihedral structure $\pi$, such that

$$
\mathcal{M}_{0, n} \subset \mathcal{M}_{0, n}^{\pi} \subset \overline{\mathcal{M}}_{0, n} .
$$

Let $z=\left(z_{1}, \ldots, z_{n}\right)$ denote the set of the $n$ marked points on the curve. In the following we will use the notation

$$
\mathcal{M}_{0, z}
$$

for $\mathcal{M}_{0, n}$. This notation allows us to distinguish $\mathcal{M}_{0, z^{\prime}}$ from $\mathcal{M}_{0, z^{\prime \prime}}$ if $z^{\prime}$ and $z^{\prime \prime}$ are two nonidentical subsets of $z$ with $k$ elements each (i.e. $z^{\prime} \neq z^{\prime \prime}$ but $\left|z^{\prime}\right|=\left|z^{\prime \prime}\right|=k$ ). Let $\pi$ denote a permutation of $(1, \ldots, n)$, which defines a dihedral structure. We may draw a regular $n$-gon, where the edges are labelled by $z_{\pi_{1}}, z_{\pi_{2}}, \ldots, z_{\pi_{n}}$ in this order. In order to keep the notation simple let us assume that $\pi=(1,2, \ldots, n)$. Then the edges are labelled by $z_{1}, z_{2}$, $\ldots, z_{n}$. A chord of the polygon connects two non-adjacent vertices and may be specified by giving the two edges preceding the two vertices in the clockwise orientation. Thus $(i, j)$ denotes the chord from the vertex between edge $z_{i}$ and $z_{i+1}$ to the vertex between the edge $z_{j}$ and $z_{j+1}$. There are

$$
\frac{1}{2} n(n-3)
$$

chords for a regular $n$-gon. We denote by $\chi(z, \pi)$ the set of all chords of the $n$-gon defined by the set $z$ and the dihedral structure $\pi$. Each chord defines a cross-ratio as follows:

$$
u_{i, j}=\frac{\left(z_{i}-z_{j+1}\right)\left(z_{i+1}-z_{j}\right)}{\left(z_{i}-z_{j}\right)\left(z_{i+1}-z_{j+1}\right)} .
$$

The cross-ratio is invariant under $\operatorname{PSL}(2, \mathbb{C})$-transformations. Each cross-ratio defines a function

$$
\mathcal{M}_{0, z} \rightarrow \mathbb{C P}^{1} \backslash\{0,1, \infty\}
$$

or equivalently

$$
\mathcal{M}_{0, z} \rightarrow \mathbb{C} \backslash\{0,1\}
$$


The set of all cross-ratios for a given dihedral structure $\pi$ defines an embedding

$$
\mathcal{M}_{0, z} \rightarrow \mathbb{C}^{n(n-3) / 2}
$$

One defines the dihedral extension $\mathcal{M}_{0, z}^{\pi}$ of $\mathcal{M}_{0, z}$ to be the Zariski closure of the image of this embedding. The Deligne-Mumford-Knudsen compactification is obtained by gluing these charts together:

$$
\overline{\mathcal{M}}_{0, z}=\bigcup_{\pi} \mathcal{M}_{0, z}^{\pi}
$$

where $\pi$ ranges over the $(n-1) ! / 2$ inequivalent dihedral structures.

\subsection{The dihedral extension}

Central to our study will be the dihedral extension $\mathcal{M}_{0, z}^{\pi}$. We recall that the construction of $\mathcal{M}_{0, z}^{\pi}$ requires the specification of a dihedral structure $\pi$ (i.e. a permutation up to cyclic permutations and reflection). We will need a few properties of the dihedral extension $\mathcal{M}_{0, z}^{\pi}[24]:$

1. The complement $\mathcal{M}_{0, z}^{\pi} \backslash \mathcal{M}_{0, z}$ is a normal crossing divisor, whose irreducible components are

$$
D_{i j}=\left\{u_{i, j}=0\right\},
$$

indexed by the chords $(i, j) \in \chi(z, \pi)$.

2. Each divisor is again a product of spaces of the same type: let us consider a chord $(i, j)$. This chord decomposes the original polygon $(z, \pi)$ into two smaller polygons, as shown in figure 2 . We denote the new edge by $z_{e}$. The set of edges for the two smaller polygons are $z^{\prime} \cup\left\{z_{e}\right\}$ and $z^{\prime \prime} \cup\left\{z_{e}\right\}$, where $z=z^{\prime} \cup z^{\prime \prime}$ and $z^{\prime} \cap z^{\prime \prime}=\emptyset$. The two smaller polygons inherit their dihedral structures $\pi^{\prime}$ and $\pi^{\prime \prime}$ from $\pi$ and the chord $(i, j)$. We have

$$
D_{i j} \cong \mathcal{M}_{0, z^{\prime} \cup\left\{z_{e}\right\}}^{\pi^{\prime}} \times \mathcal{M}_{0, z^{\prime \prime} \cup\left\{z_{e}\right\}}^{\pi^{\prime \prime}}
$$

\subsection{The associahedron}

Let us now consider the space of real points. For a given set $z$ and dihedral structure $\pi$ we set

$$
X_{0, z}^{\pi}=\left\{u_{i, j}>0:(i, j) \in \chi(z, \pi)\right\}
$$

and

$$
\bar{X}_{0, z}^{\pi}=\left\{u_{i, j} \geq 0:(i, j) \in \chi(z, \pi)\right\} .
$$

One has

$$
\mathcal{M}_{0, n}(\mathbb{R})=\bigsqcup_{\pi} X_{0, z}^{\pi}
$$

where $\pi$ ranges again over the $(n-1) ! / 2$ inequivalent dihedral structures. 
For a given set $z$ and dihedral structure $\pi$ the cell $\bar{X}_{0, z}^{\pi}$ is called a Stasheff polytope or associahedron [25-28]. The associahedron has the properties

1. Its facets (i.e. codimension one faces)

$$
F_{i j}=\left\{u_{i, j}=0\right\},
$$

are indexed by the chords $(i, j) \in \chi(z, \pi)$.

2. From eq. (3.14) it follows that each facet is a product

$$
F_{i j}=\bar{X}_{0, z^{\prime} \cup\left\{z_{e}\right\}}^{\pi^{\prime}} \times \bar{X}_{0, z^{\prime \prime} \cup\left\{z_{e}\right\}}^{\pi^{\prime \prime}}
$$

3. Two facets $F_{i j}$ and $F_{k l}$ meet if and only if the chords $(i, j)$ and $(k, l)$ do not cross.

4. Faces of codimension $k$ are given by sets of $k$ non-crossing chords. In particular, the set of vertices of $\bar{X}_{0, z}^{\pi}$ are in one-to-one correspondence with the set of triangulations of the $n$-gon defined by the set $z$ and the dihedral structure $\pi$.

Properties (1) and (2) are the analogues of eq. (3.13) and eq. (3.14), respectively.

\subsection{Coordinates on $\mathcal{M}_{0, z}^{\pi}$}

Let us now fix a dihedral structure $\pi$. Without loss of generality we may take the cyclic order to be $(1,2, \ldots, n)$. Let us consider a chord from $\chi(z, \pi)$. Due to cyclic invariance we may limit ourselves to chords of the form $(i, n)$. With the gauge choice $z_{1}=0, z_{n-1}=1$ and $z_{n}=\infty$ we have

$$
u_{2, n}=\frac{z_{2}}{z_{3}}, \quad \ldots \quad u_{(n-3), n}=\frac{z_{n-3}}{z_{n-2}}, \quad u_{(n-2), n}=z_{n-2},
$$

and hence

$$
z_{i}=\prod_{j=i}^{n-2} u_{j, n}, \quad i \in\{2, \ldots, n-2\} .
$$

Thus we may use as coordinates on $\mathcal{M}_{0, z}^{\pi}$ instead of the $(n-3)$ coordinates $\left(z_{2}, \ldots, z_{n-2}\right)$ the $(n-3)$ cross-ratios $\left(u_{2, n}, \ldots, u_{n-2, n}\right)$. We have

$$
d^{n-3} z=\left(\prod_{j=3}^{n-2} u_{j, n}^{j-2}\right) d^{n-3} u
$$

Let us now fix $i_{0} \in\{2, \ldots, n-2\}$. We will study the limit $u_{i_{0}, n} \rightarrow 0$. The chord $\left(i_{0}, n\right)$ splits the polygon into two smaller polygons. We set $z^{\prime}=\left(z_{1}, z_{2}, \ldots, z_{i_{0}}\right)$ and $z^{\prime \prime}=\left(z_{i_{0}+1}, \ldots, z_{n}\right)$. As before we label the new edge by $z_{e}$. One of the two smaller polygons has the edges $z^{\prime} \cup\left\{z_{e}\right\}$ and the dihedral structure $\pi^{\prime}=\left(1,2, \ldots, i_{0}, e\right)$, the other smaller polygon has the edges $z^{\prime \prime} \cup\left\{z_{e}\right\}$ and the dihedral structure $\pi^{\prime \prime}=\left(e, i_{0}+1, i_{0}+2, \ldots, n\right)$. In the limit $u_{i_{0}, n} \rightarrow 0$ we have

$$
\lim _{u_{i_{0}, n} \rightarrow 0} u_{i, j}=1
$$

for any chord $(i, j) \in \chi(z, \pi)$ which crosses the chord $\left(i_{0}, n\right) \in \chi(z, \pi)$. 
Let us express the cyclic factor $C(\pi, z)$ in the new variables $u_{j, n}$. We have

$$
\lim _{z_{n} \rightarrow \infty} z_{n}^{2} C(\pi, z)=\prod_{j=2}^{n-2} u_{j, n}^{1-j}\left(u_{j, n}-1\right)^{-1} .
$$

Thus

$$
C(\pi, z) \frac{d^{n} z}{d \omega}=\left(\prod_{j=2}^{n-2} \frac{1}{u_{j, n}\left(u_{j, n}-1\right)}\right) d^{n-3} u
$$

\section{The scattering forms}

In this section we study the scattering forms

$$
\begin{aligned}
\Omega_{\text {scattering }}^{\text {cyclic }}(\sigma, z) & =C(\sigma, z) \frac{d^{n} z}{d \omega}, \\
\Omega_{\text {scattering }}^{\text {pol }}(p, \varepsilon, z) & =E(p, \varepsilon, z) \frac{d^{n} z}{d \omega} .
\end{aligned}
$$

The polarisation factor $E(p, \varepsilon, z)$ will be defined below. These scattering forms have interesting properties:

1. The scattering forms are $\operatorname{PSL}(2, \mathbb{C})$-invariant.

2. The twisted intersection numbers give the amplitudes for the bi-adjoint scalar theory (cyclic, cyclic), Yang-Mills theory (cyclic,polarisation) and gravity (polarisation,polarisation).

3. The only singularities of the scattering forms are on the divisor $\overline{\mathcal{M}}_{0, n} \backslash \mathcal{M}_{0, n}$.

4. The singularities are logarithmic.

5. The residues at the singularities factorise into two scattering forms of lower points.

In addition, the scattering forms have specific properties under permutations: the scattering form $\Omega_{\text {scattering }}^{\text {cyclic }}(\sigma, z)$ is invariant under cyclic permutations and satisfies the Kleiss-Kuijf relations [29]. The scattering form $\Omega_{\mathrm{scattering}}^{\mathrm{pol}}(p, \varepsilon, z)$ is permutation-invariant.

The most remarkable property is certainly the factorisation of the residues. Please note that we do not require a particular kinematic limit in the momenta variables, like one momentum soft or $s_{I} \rightarrow 0$ for a subset $I$ of $\{1,2, \ldots, n\}$. The factorisation of the residues of the scattering forms holds for any momentum configuration $p$. The factorisation of the residues is illustrated in figure 3. We may iterate this procedure and take multiple residues. Taking the maximal number of residues produces a 0 -form and corresponds to a triangulation of the $n$-gon. Each triangulation defines a dual graph $G$ with trivalent vertices, as shown in figure 4, and conversely, every dual graph with trivalent vertices defines a triangulation. The $(n-3)$-fold maximal residue is given by the numerator of the dual graph. This graph factorises into three-valent vertices, where a sum over internal polarisations and particle flavours is understood. 



Figure 3. We may visualise a scattering form on $\mathcal{M}_{0, n}^{\pi}$ as shown in the left figure: the dihedral structure defines an $n$-gon, each external line of the scattering process crosses one edge of the $n$-gon. In the limit $u_{i_{0}, n} \rightarrow 0$ the residue factorises into two scattering forms of lower points, as shown in the right figure.

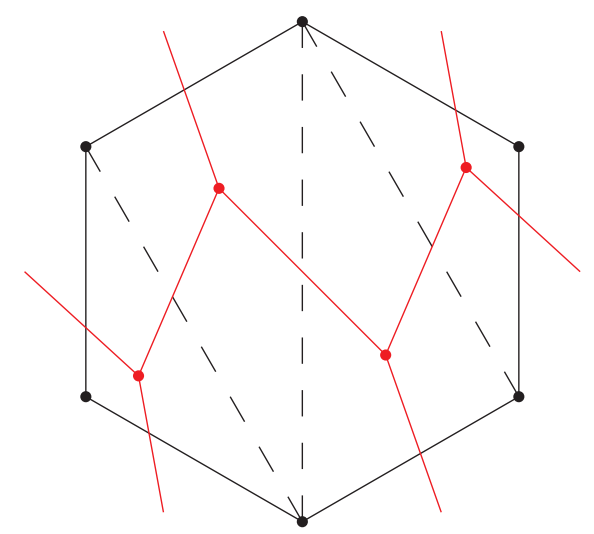

Figure 4. A hexagon with a triangulation, given by the dashed lines. The dual graph is shown in red.

Property 2 has been shown recently by Mizera [5]. We believe that properties $3-5$ for $\Omega_{\text {scattering }}^{\text {cyclic }}$ are known to experts in the field, the fact that the singularities of $\Omega_{\text {scattering }}^{\text {cyclic }}$ are logarithmic is shown in [6]. These properties can also be inferred from the conference talks [30,31]. The essential new ingredient of this paper is that $\Omega_{\text {scattering }}^{\mathrm{pol}}$ can be defined with the same properties.

\subsection{The cyclic scattering form}

Let us start with

$$
\Omega_{\text {scattering }}^{\text {cyclic }}(\sigma, z)=C(\sigma, z) \frac{d^{n} z}{d \omega} .
$$

$\operatorname{PSL}(2, \mathbb{C})$-invariance is straightforward. Property $(2)$ is the statement that with

$$
\Omega=\Omega_{\text {scattering }}^{\text {cyclic }}(\sigma, z), \tilde{\Omega}=\Omega_{\text {scattering }}^{\text {cyclic }}(\tilde{\sigma}, z)
$$

one has

$$
m_{n}(\sigma, \tilde{\sigma}, p)=i \oint_{\mathcal{C}} d \Omega_{\mathrm{CHY}} C(\sigma, z) C(\tilde{\sigma}, z)=i(\Omega, \tilde{\Omega})_{\eta}
$$




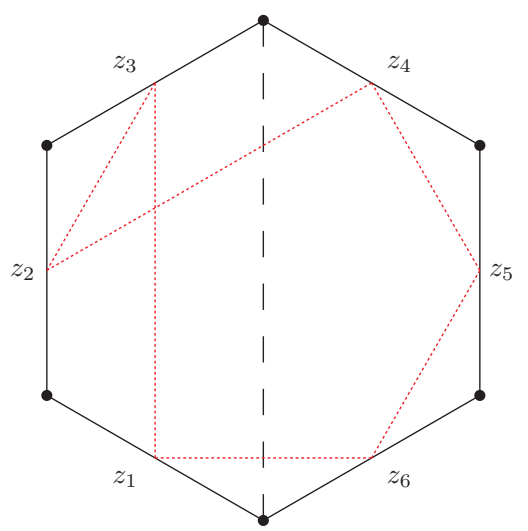

Figure 5. A hexagon with the dihedral structure $\pi=(1,2,3,4,5,6)$. The dashed line shows the chord $(3,6)$. The dotted red lines show the bonds corresponding to the cyclic factor $C(\sigma, z)$ for $\sigma=(1,3,2,4,5,6)$. The bonds $\left(z_{2}-z_{4}\right)$ and $\left(z_{6}-z_{1}\right)$ cross the chord $(3,6)$.

where $(\Omega, \tilde{\Omega})_{\eta}$ denotes the twisted intersection number of $\Omega$ and $\tilde{\Omega}$ with twist $\eta$ (defined in eq. (2.36)). The last equality has been shown recently by Mizera [5].

Property (3) is also clear: the cyclic factor $C(\sigma, z)$ becomes singular whenever two $z$ 's adjacent in the cyclic order $\sigma$ coincide:

$$
z_{\sigma_{i}}=z_{\sigma_{i+1}}
$$

These points are on the divisor $\overline{\mathcal{M}}_{0, n} \backslash \mathcal{M}_{0, n}$.

In order to show points (4) and (5) we have to work a little bit more. We consider the dihedral extension $\mathcal{M}_{0, n}^{\pi}$. Without loss of generality we may take $\pi=(1,2, \ldots, n)$. Further let $i_{0} \in\{2, \ldots, n-2\}$. The variable $i_{0}$ defines a chord $\left(i_{0}, n\right)$ and a cross-ratio $u_{i_{0}, n}$. We now consider the behaviour of $\Omega_{\text {scattering }}^{\text {cyclic }}(\sigma, z)$ in the limit $u_{i_{0}, n} \rightarrow 0$. Since we fixed $\pi=(1,2, \ldots, n)$, the other permutation $\sigma=\left(\sigma_{1}, \ldots, \sigma_{n}\right)$ is arbitrary. Let us call

$$
z_{\sigma_{i}}-z_{\sigma_{j}}
$$

a bond connecting the edges $z_{\sigma_{i}}$ and $z_{\sigma_{j}}$. This is illustrated in figure 5 . We say that a bond $\left(z_{\sigma_{i}}-z_{\sigma_{j}}\right)$ crosses the chord $\left(i_{0}, n\right)$ if

$$
\left(\sigma_{i} \in\left\{1, \ldots, i_{0}\right\} \text { and } \sigma_{j} \in\left\{i_{0}+1, \ldots, n\right\}\right) \text { or }\left(\sigma_{j} \in\left\{1, \ldots, i_{0}\right\} \text { and } \sigma_{i} \in\left\{i_{0}+1, \ldots, n\right\}\right) \text {. }
$$

Now let us look at the cyclic factor

$$
C(\sigma, z)=\frac{1}{\left(z_{\sigma_{1}}-z_{\sigma_{2}}\right) \ldots\left(z_{\sigma_{n-1}}-z_{\sigma_{n}}\right)\left(z_{\sigma_{n}}-z_{\sigma_{1}}\right)}
$$

We are interested in the number of bonds crossing the chord $\left(i_{0}, n\right)$. It is easy to see that this number must be even and that there are at least two bonds crossing the chord $\left(i_{0}, n\right)$. The maximal number of bonds crossing the chord $\left(i_{0}, n\right)$ is given by

$$
2 \min \left(i_{0}, n-i_{0}\right)
$$


We call $\sigma$ and $\pi$ equivalent with respect to the chord $\left(i_{0}, n\right)$

$$
\sigma \sim{ }_{\left(i_{0}, n\right)} \pi
$$

if there are exactly two bonds in $C(\sigma, z)$ crossing the chord $\left(i_{0}, n\right)$. It is not too difficult to see that this is the case if and only if $\sigma$ can be written (after a suitable cyclic permutation) in the form

$$
\sigma=\left(\sigma_{1}^{\prime}, \ldots, \sigma_{i_{0}}^{\prime}, \sigma_{i_{0}+1}^{\prime \prime}, \ldots, \sigma_{n}^{\prime \prime}\right)
$$

with

$$
\sigma_{j}^{\prime} \in\left\{1, \ldots, i_{0}\right\} \quad \text { and } \quad \sigma_{k}^{\prime \prime} \in\left\{i_{0}+1, \ldots, n\right\} .
$$

If $\sigma$ and $\pi$ are equivalent with respect to the chord $\left(i_{0}, n\right)$ we define the induced dihedral structures $\sigma^{\prime}$ and $\sigma^{\prime \prime}$ to be

$$
\sigma^{\prime}=\left(\sigma_{1}^{\prime}, \ldots, \sigma_{i_{0}}^{\prime}, e\right), \sigma^{\prime \prime}=\left(e, \sigma_{i_{0}+1}^{\prime \prime}, \ldots, \sigma_{n}^{\prime \prime}\right) .
$$

These considerations are helpful to answer the following question: how many factors of $u_{i_{0}, n}$ does the cyclic factor $C(\sigma, z)$ produce, if we change the variables from the $z_{j}$ 's to the cross-ratios $u_{j, n}$ 's? A bond

$$
z_{\sigma_{i}}-z_{\sigma_{j}}
$$

gives a factor $u_{i_{0}, n}$ if $\sigma_{i}, \sigma_{j} \in\left\{1, \ldots, i_{0}\right\}$ and no factor of $u_{i_{0}, n}$ in all other cases. Thus $C(\sigma, z)$ has a factor

$$
u_{i_{0}, n}^{\frac{n_{\text {cross }}}{2}-i_{0}},
$$

where $n_{\text {cross }}$ denotes the number of bonds crossing the chord $\left(i_{0}, n\right)$. We obtain the maximal (negative) power of $u_{i_{0}, n}$

$$
u_{i_{0}, n}^{1-i_{0}},
$$

if $\sigma$ is equivalent to $\pi$ with respect to the chord $\left(i_{0}, n\right)$. In all other cases we obtain fewer powers of $1 / u_{i_{0}, n}$. Combined with the factor

$$
u_{i_{0}, n}^{i_{0}-2}
$$

from the measure we obtain a single pole $1 / u_{i_{0}, n}$ if $\sigma$ is equivalent to $\pi$ with respect to the chord $\left(i_{0}, n\right)$, and no pole in all other cases. This proves property (4).

Let us now look at the residues. If $\sigma \nsim_{\left(i_{0}, n\right)} \pi$ there aren't enough negative powers of $u_{i_{0}, n}$ from the cyclic factors $C(\sigma, z)$ to produce a non-zero residue. If $\sigma \sim_{\left(i_{0}, n\right)} \pi$ the scattering form $\Omega_{\text {scattering }}^{\text {cyclic }}(\sigma, z)$ has a single pole at $u_{i_{0}, n}=0$. The chord $\left(i_{0}, n\right)$ divides the polygon with dihedral structure $\pi$ into two smaller polygons with dihedral structures $\pi^{\prime}$ and $\pi^{\prime \prime}$, respectively. As $\sigma$ is equivalent to $\pi$ with respect to the chord $\left(i_{0}, n\right)$ we have the induced structures $\sigma^{\prime}$ and $\sigma^{\prime \prime}$. On the polygon with dihedral structure $\pi^{\prime}$ we use the coordinates $u_{2, n}, \ldots, u_{i_{0}-1, n}$, on the polygon with dihedral structure $\pi^{\prime \prime}$ we use the coordinates $u_{i_{0}+1, n}, \ldots, u_{n-2, n}$. We denote by $Y$ be the hypersurface given by $u_{i_{0}, n}=0$. 
After cancelling common factors of $\left(u_{i_{0}+1, n} u_{i_{0}+2, n} \ldots u_{n-2, n}\right)$ from the numerator and the denominator one finds

$$
\operatorname{Res}_{Y} \Omega_{\text {scattering }}^{\text {cyclic }}(\sigma, z)= \begin{cases}(-1)^{i_{0}-1} \Omega_{\text {scattering }}^{\text {cyclic }}\left(\sigma^{\prime}, z\right) \wedge \Omega_{\text {scattering }}^{\text {cyclic }}\left(\sigma^{\prime \prime}, z\right) & \text { if } \sigma \sim_{\left(i_{0}, n\right)} \pi \\ 0 & \text { otherwise }\end{cases}
$$

Thus we see that the residue factorises into two scattering forms of lower points. The factorisation of the residue is illustrated in figure 3 .

The scattering form $\Omega_{\text {scattering }}^{\text {cyclic }}(\sigma, z)$ inherits the transformation properties under permutations from the cyclic factor $C(\sigma, z)$. It is invariant under cyclic permutations and satisfies the Kleiss-Kuijf relations [29]. Let $\alpha=\left(\alpha_{1}, \ldots, \alpha_{j}\right)$ be a permutation of $(2, \ldots, j+1)$ and $\beta=\left(\beta_{1}, \ldots, \beta_{n-2-j}\right)$ a permutation of $(j+2, \ldots, n-1)$. Then

$$
\Omega_{\text {scattering }}^{\text {cyclic }}((1, \alpha, n, \beta), z)=(-1)^{n-2-j} \sum_{\sigma \in \alpha \amalg \beta^{T}} \Omega_{\text {scattering }}^{\text {cyclic }}((1, \sigma, n), z),
$$

where $\alpha \uplus \beta^{T}$ denotes the set of all shuffles of $\alpha$ with $\beta^{T}=\left(\beta_{n-2-j}, \ldots, \beta_{1}\right)$, i.e. the set of all permutations of the elements of $\alpha$ and $\beta^{T}$, which preserve the relative order of the elements of $\alpha$ and of the elements of $\beta^{T}$.

\subsubsection{Examples}

Let us give a few examples. We consider $\mathcal{M}_{0, n}^{\pi}$ with $\pi=(1,2, \ldots, n)$. We fix $z_{1}=0$, $z_{n-1}=1$ and $z_{n}=\infty$. We have

$$
\frac{d^{n} z}{d \omega}=z_{n}\left(z_{n}-1\right) d z_{2} \wedge d z_{3} \wedge \ldots \wedge d z_{n-2} .
$$

Let us first discuss the case $n=3$. For three particles the scattering form $\Omega_{\text {scattering }}^{\text {cyclic }}(\sigma, z)$ is a 0 -form. On $\mathcal{M}_{0,3}^{\pi}$ the scattering form is given by

$$
\Omega_{\text {scattering }}^{\text {cyclic }}(\sigma, z)= \pm 1 \text {, }
$$

depending on whether $\sigma$ is an even or odd permutation of $\pi=(1,2,3)$.

For $n=4$ and $\sigma=\pi=(1,2,3,4)$ we obtain

$$
\Omega_{\text {scattering }}^{\text {cyclic }}(\sigma, z)=\frac{d z_{2}}{z_{2}\left(z_{2}-1\right)}=\frac{d u_{2,4}}{u_{2,4}\left(u_{2,4}-1\right)} .
$$

The residue at $u_{2,4}=0$ is given by

$$
\operatorname{Res}_{u_{2,4}=0} \Omega_{\text {scattering }}^{\text {cyclic }}=-1 \text {. }
$$

Let us now consider the case $n=5$. For $\sigma=\pi=(1,2,3,4,5)$ we obtain

$$
\Omega_{\text {scattering }}^{\text {cyclic }}(\sigma, z)=\frac{d z_{2} \wedge d z_{3}}{z_{2}\left(z_{2}-z_{3}\right)\left(z_{3}-1\right)}=\frac{d u_{2,5}}{u_{2,5}\left(u_{2,5}-1\right)} \wedge \frac{d u_{3,5}}{u_{3,5}\left(u_{3,5}-1\right)} \text {. }
$$


We have the residues

$$
\operatorname{Res}_{u_{2,5}=0} \Omega_{\text {scattering }}^{\text {cyclic }}=-\frac{d u_{3,5}}{u_{3,5}\left(u_{3,5}-1\right)}, \operatorname{Res}_{u_{3,5}=0} \Omega_{\text {scattering }}^{\text {cyclic }}=\frac{d u_{2,5}}{u_{2,5}\left(u_{2,5}-1\right)} .
$$

For general $n$ we have for $\sigma=\pi=(1, \ldots, n)$

$$
\begin{aligned}
\Omega_{\text {scattering }}^{\text {cyclic }}(\sigma, z) & =\frac{d z_{2} \wedge \ldots \wedge d z_{n-2}}{z_{2}\left(z_{2}-z_{3}\right) \ldots\left(z_{n-3}-z_{n-2}\right)\left(z_{n-2}-1\right)} \\
& =\frac{d u_{2, n}}{u_{2, n}\left(u_{2, n}-1\right)} \wedge \ldots \wedge \frac{d u_{n-2, n}}{u_{n-2, n}\left(u_{n-2, n}-1\right)} .
\end{aligned}
$$

For the residues we find

$$
\begin{aligned}
\operatorname{Res}_{u_{i_{0}, n}=0} \Omega_{\text {scattering }}^{\text {cyclic }}(\sigma, z)= & (-1)^{i_{0}-1} \frac{d u_{2, n}}{u_{2, n}\left(u_{2, n}-1\right)} \wedge \ldots \wedge \frac{d u_{i_{0}-1, n}}{u_{i_{0}-1, n}\left(u_{i_{0}-1, n}-1\right)} \\
& \wedge \frac{d u_{i_{0}+1, n}}{u_{i_{0}+1, n}\left(u_{i_{0}+1, n}-1\right)} \wedge \ldots \wedge \frac{d u_{n-2, n}}{u_{n-2, n}\left(u_{n-2, n}-1\right)} .
\end{aligned}
$$

\subsection{The polarisation scattering form}

In this paragraph we define and study the scattering form

$$
\Omega_{\mathrm{scattering}}^{\text {pol }}(p, \varepsilon, z)=E(p, \varepsilon, z) \frac{d^{n} z}{d \omega} .
$$

This scattering form involves the polarisation factor $E(p, \varepsilon, z)$. Originally, the polarisation factor $E(p, \varepsilon, z)$ is defined in terms of the reduced Pfaffian

$$
\frac{(-1)^{i+j}}{2\left(z_{i}-z_{j}\right)} \operatorname{Pf} \Psi_{i j}^{i j}
$$

where $\Psi_{i j}^{i j}$ is the $(2 n-2) \times(2 n-2)$-matrix obtained from the $(2 n) \times(2 n)$-matrix $\Psi$ by deleting the rows and columns $i$ and $j$ (with $1 \leq i<j \leq n$ ). It can be shown that this definition is independent of the choice of $i$ and $j$ if

1. the momenta are on-shell: $p_{j}^{2}=0$,

2. the polarisations are transverse: $\varepsilon_{j} \cdot p_{j}=0$,

3. the variables $z$ are solutions of the scattering equations: $f_{j}(z, p)=0$.

We will need to relax all three conditions. The requirement to relax condition (3) is obvious: we want to study the polarisation factor $E(p, \varepsilon, z)$ on the full moduli space $\overline{\mathcal{M}}_{0, n}$ and not just on a zero-dimensional sub-variety defined by the solutions of the scattering equations. The requirements to relax conditions (1) and (2) become apparent once we start to discuss the structure of possible factorisations. Let us choose a dihedral structure, which we take without loss of generality as $\pi=(1,2, \ldots, n)$ and pick $i_{0} \in\{2, \ldots, n-2\}$. This defines a chord $\left(i_{0}, n\right)$, which divides the original $n$-gon into two smaller polygons. We set

$$
\begin{aligned}
p^{\prime} & =\left(p_{1}, p_{2}, \ldots, p_{i_{0}}, p_{i_{0}+1}+p_{i_{0}+2}+\ldots+p_{n}\right), \\
p^{\prime \prime} & =\left(p_{1}+p_{2}+\ldots+p_{i_{0}}, p_{i_{0}+1}, p_{i_{0}+2}, \ldots, p_{n}\right) .
\end{aligned}
$$


Note that the momenta of $p^{\prime}$ and $p^{\prime \prime}$ are no longer necessarily on the mass-shell. However, they satisfy momentum conservation. Thus in general we have

$$
p_{j}^{2} \neq 0 \text {. }
$$

For the polarisation vectors we set

$$
\begin{aligned}
\varepsilon^{\prime} & =\left(\varepsilon_{1}, \varepsilon_{2}, \ldots, \varepsilon_{i_{0}}, \varepsilon_{e}\right), \\
\varepsilon^{\prime \prime} & =\left(\varepsilon_{e}^{*}, \varepsilon_{i_{0}+1}, \varepsilon_{i_{0}+2}, \ldots, \varepsilon_{n}\right),
\end{aligned}
$$

where we introduced for the new edge defined by the chord $\left(i_{0}, n\right)$ a new polarisation vector $\varepsilon_{e}$. In four space-time dimensions massless gauge bosons have two physical polarisations, which we may take as

$$
\varepsilon_{\mu}^{+}(p, q)=-\frac{\left\langle p+\left|\gamma_{\mu}\right| q+\right\rangle}{\sqrt{2}\langle p-\mid q+\rangle}, \varepsilon_{\mu}^{-}(p, q)=\frac{\left\langle p-\left|\gamma_{\mu}\right| q-\right\rangle}{\sqrt{2}\langle p+\mid q-\rangle}
$$

where $p$ is the momentum of the gauge boson and $q$ is an arbitrary light-like reference momentum. The polarisation sum over the physical polarisation is given by

$$
\sum_{\lambda \in\{+,-\}}\left(\varepsilon_{\mu}^{\lambda}\right)^{*} \varepsilon_{\nu}^{\lambda}=-g_{\mu \nu}+\frac{p_{\mu} q_{\nu}}{p \cdot q}+\frac{q_{\mu} p_{\nu}}{p \cdot q} .
$$

On both sides we have a $4 \times 4$-matrix (in $\mu, \nu$ ) of rank 2 . In order to obtain a matrix of full rank, let us supplement the two physical polarisations $\varepsilon^{+}$and $\varepsilon^{-}$by two un-physical polarisations $\varepsilon^{0}$ and $\varepsilon^{\overline{0}}$, sucht that

$$
\sum_{\lambda \in\{+,-, 0, \overline{0}\}}\left(\varepsilon_{\mu}^{\lambda}\right)^{*} \varepsilon_{\nu}^{\lambda}=-g_{\mu \nu} .
$$

In $D$ space-time dimensions we have $(D-2)$ physical polarisations and 2 un-physical polarisations. In this paper we will never need to specify the explicit expressions for the polarisation vectors. We only need to assume that in arbitrary space-time dimensions there is a set of (physical and un-physical) polarisation vectors indexed by $\lambda$ such that

$$
\sum_{\lambda}\left(\varepsilon_{\mu}^{\lambda}\right)^{*} \varepsilon_{\nu}^{\lambda}=-g_{\mu \nu}
$$

With this generalisation we now have in general

$$
\varepsilon_{j} \cdot p_{j} \neq 0
$$

Analogously we may introduce (un-physical) polarisations $\varepsilon_{\mu \nu}^{\lambda}$ for the auxiliary tensor particle appearing in eq. (2.17) and eq. (2.18), such that

$$
\sum_{\lambda}\left(\varepsilon_{\mu \nu}^{\lambda}\right)^{*} \varepsilon_{\rho \sigma}^{\lambda}=-\frac{1}{2} p^{2}\left(g_{\mu \rho} g_{\nu \sigma}-g_{\mu \sigma} g_{\nu \rho}\right) .
$$

We may iterate this procedure for all new vertices appearing in the effective Lagrangian of eq. (2.21). As an example we discuss a five-valent vertex, appearing in $\mathcal{L}^{(5)}$. This vertex has 



Figure 6. The left picture shows the tree structure underlying a five-valent vertex from $\mathcal{L}^{(5)}$. For the intermediate propagators we may introduce auxiliary particles, shown in green and red in the middle picture. Introducing quantum numbers $g, \bar{g}$ and $r, \bar{r}$ such that the new auxiliary particles propagate only from $g$ to $\bar{g}$ and from $r$ to $\bar{r}$ ensures that the new three-valent vertices (shown in the right picture) recombine only into the original five-valent vertex.

an underlying tree structure as shown in the left picture of figure 6. (For five points there is only one possible underlying tree structure.) We may introduce two auxiliary particles for the intermediate edges, as shown in the middle picture of figure 6 . We may associate quantum numbers to these particles, say $g$ and $r$, such that the propagation is only from $g$ to $\bar{g}$ and from $r$ to $\bar{r}$. In this way we obtain three new three-valent vertices, as shown in the right picture of figure 6 . Note that the introduction of the quantum numbers $g$ and $r$ ensures, that the new auxiliary vertices and propagators recombine only into the original five-valent vertex.

In the following we will write

$$
\sum_{f, \lambda}
$$

for a sum over particle species $f$ (gauge boson and auxiliary particles) and the corresponding polarisations $\lambda$. By a factorisation of a numerator $N(G)$ corresponding to a graph $G=\left(G_{1}, G_{2}\right)$ we understand a factorisation of the form

$$
N(G)=\sum_{f, \lambda} N\left(G_{1}\right) N\left(G_{2}\right)
$$

where the sum is over all particles and polarisations corresponding to the edge connecting the sub-graphs $G_{1}$ and $G_{2}$.

The reduced Pfaffian has been studied quite extensively in the literature [32-41]. The expression given by the reduced Pfaffian is not very well suited to generalise towards off-shell momenta, unphysical polarisation or away from the solutions of the scattering equations. Appendix A.2 provides further details on this point. In order to construct the polarisation factor $E(p, \varepsilon, z)$ in the general off-shell case with unphysical polarisations and away from the solutions of the scattering equations we proceed along a different way. As polarisation factor we may take

$$
E(p, \varepsilon, z)=\sum_{\kappa \in S_{n-2}^{(i, j)}} C(\kappa, z) N_{\mathrm{comb}}^{\mathrm{BCJ}}(\kappa),
$$

where $i, j \in\{1, \ldots, n\}, i \neq j$ and $\kappa$ is a permutation of $\{1, \ldots, n\}$ with $\kappa_{1}=i$ and $\kappa_{n}=j$. The set of all these permutations is denoted by $S_{n-2}^{(i, j)}$. The BCJ-numerators $N_{\text {comb }}^{\text {BCJ }}(\kappa)$ have been defined in eq. (2.28). Eq. (4.40) is clearly invariant under the $(n-2)$ ! permutations of $\{1, \ldots, n\} \backslash\{i, j\}$. It can be shown that eq. (4.40) is actually invariant under all $n$ ! 
permutations of $\{1, \ldots, n\}$ and therefore independent of the choice of $i$ and $j$. A proof is given in appendix C. Our standard choice will be $i=1$ and $j=n$, yielding

$$
\begin{aligned}
\Omega_{\text {scattering }}^{\text {pol }}(p, \varepsilon, z) & =E(p, \varepsilon, z) \frac{d^{n} z}{d \omega}, \\
E(p, \varepsilon, z) & =\sum_{\kappa \in S_{n-2}^{(1, n)}} C(\kappa, z) N_{\mathrm{comb}}^{\mathrm{BCJ}}(\kappa),
\end{aligned}
$$

where the sum is now over all $(n-2)$ ! permutations of $\{2, \ldots,(n-1)\}$, keeping $\kappa_{1}=1$ and $\kappa_{n}=n$ fixed.

Let us now discuss the properties of $\Omega_{\text {scattering }}^{\text {pol }}$. As already mentioned, the polarisation factor $E(p, \varepsilon, z)$ is permutation-invariant, hence $\Omega_{\text {scattering }}^{\text {pol }}$ is permutation-invariant.

Eq. (4.41) is an expansion in cyclic factors. This has three immediate implications. First of all, PSL $(2, \mathbb{C})$-invariance is manifest. Secondly, it follows that the only singularities

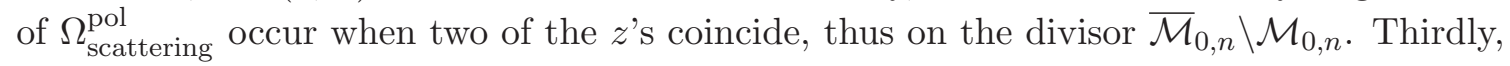
from the discussion in section 4.1 it follows that all singularities are logarithmic.

It is a little bit harder to show the remaining two properties: firstly, we have to show that $E(p, \varepsilon, z)$ as defined by eq. (4.41) agrees with the reduced Pfaffian in the on-shell limit with physical polarisations and on the sub-variety defined by the scattering equations. Secondly, we have to establish the factorisation of the residues.

Let us start with the equivalence of $E(p, \varepsilon, z)$ with the reduced Pfaffian for on-shell momenta, physical polarisations and on the sub-variety defined by the scattering equations. Let us therefore assume on-shell momenta and physical polarisations. $E(p, \varepsilon, z)$ agrees with the reduced Pfaffian for all $(n-3)$ ! solutions of the scattering equations $z^{(j)}$ if and only if

$$
i \oint_{\mathcal{C}} d \Omega_{\mathrm{CHY}} C(\sigma, z) E(p, \varepsilon, z)
$$

reproduces the Yang-Mills amplitude $A_{n}(\sigma, p, \varepsilon)$ for a basis of $(n-3)$ ! cyclic orders $\sigma$. Since $E(p, \varepsilon, z)$ is permutation-invariant it suffices to check eq. (4.42) for one cyclic order $\sigma$. It is convenient to take $\sigma=(1,2, \ldots, n)$. From eq. (2.20) we have

$$
A_{n}(\sigma, p, \varepsilon)=i(-1)^{n-3} \sum_{G \in \mathcal{T}_{n}(\sigma)} N^{\mathrm{BCJ}}(G) \prod_{e \in E(G)} \frac{1}{s_{e}} .
$$

Working out eq. (4.42) we obtain

$$
\begin{aligned}
& i \oint_{\mathcal{C}} d \Omega_{\mathrm{CHY}} C(\sigma, z) E(p, \varepsilon, z)= \\
& i(-1)^{n-3} \sum_{G \in \mathcal{T}_{n}(\sigma)}\left(\prod_{e \in E(G)} \frac{1}{s_{e}}\right) \sum_{\tilde{\sigma} \in \mathrm{CO}(G)} \sum_{\kappa \in S_{n-2}^{(1, n)}} \delta_{\tilde{\sigma}, \kappa}(-1)^{n_{\mathrm{flip}}(\sigma, \kappa)} N_{\mathrm{comb}}^{\mathrm{BCJ}}(\kappa),
\end{aligned}
$$

where we used a formula similar to eq. (2.8) to exchange summation orders and where we used eq. (2.15). The symbol $\delta_{\sigma, \sigma^{\prime}}$ equals 1 if $\sigma=\sigma^{\prime}$ as $n$-tuples and zero otherwise. Let us 
denote

$$
\hat{N}^{\mathrm{BCJ}}(G)=\sum_{\tilde{\sigma} \in \mathrm{CO}(G)} \sum_{\kappa \in S_{n-2}^{(1, n)}} \delta_{\tilde{\sigma}, \kappa}(-1)^{n_{\mathrm{flip}}(\sigma, \kappa)} N_{\mathrm{comb}}^{\mathrm{BCJ}}(\kappa) .
$$

Eq. (4.43) and eq. (4.44) are certainly equal if

$$
\hat{N}^{\mathrm{BCJ}}(G)=N^{\mathrm{BCJ}}(G) .
$$

Actually, it would be sufficient if $\hat{N}^{\mathrm{BCJ}}(G)$ and $N^{\mathrm{BCJ}}(G)$ are related by a generalised gauge transformation, i.e.

$$
\sum_{G \in \mathcal{T}_{n}(\sigma)} \frac{\hat{N}^{\mathrm{BCJ}}(G)-N^{\mathrm{BCJ}}(G)}{\prod_{e \in E(G)} s_{e}}=0 .
$$

However, for the case at hand one can show that the stronger condition of eq. (4.46) holds. In fact, $\hat{N}^{\mathrm{BCJ}}(G)$ is nothing else than the reduction of the numerator $N^{\mathrm{BCJ}}(G)$ to the basis of BCJ-numerators $N_{\text {comb }}^{\mathrm{BCJ}}(\kappa)$ with $\kappa \in S_{n-2}^{(1, n)}$ by repeated use of eq. (2.26). The details are given in appendix B. Having established that the definition of the polarisation factor in eq. (4.40) agrees with the reduced Pfaffian for on-shell momenta, physical polarisations and on the sub-variety defined by the scattering equations, we may use the expression of eq. (4.40) also for the gravity amplitudes.

Let us now look at the factorisation of the residues. We consider the dihedral extension $\mathcal{M}_{0, n}^{\pi}$. Without loss of generality we may take $\pi=(1,2, \ldots, n)$. Further let $i_{0} \in\{2, \ldots, n-$ $2\}$. The variable $i_{0}$ defines a chord $\left(i_{0}, n\right)$ and a cross-ratio $u_{i_{0}, n}$. We now consider the behaviour of $\Omega_{\text {scattering }}^{\text {pol }}(p, \varepsilon, z)$ in the limit $u_{i_{0}, n} \rightarrow 0$. Let us denote by $Y$ the hypersurface given by $u_{i_{0}, n}=0$. The chord $\left(i_{0}, n\right)$ divides the $n$-gon defined by $\pi$ into two smaller polygons. We denote by $p^{\prime}$ and $\varepsilon^{\prime}$ the induced data of one of the two smaller polygons, and by $p^{\prime \prime}$ and $\varepsilon^{\prime \prime}$ the induced data of the other smaller polygon. The new momenta $p^{\prime}$ and $p^{\prime \prime}$ are defined by eq. (4.29), the new polarisations $\varepsilon^{\prime}$ and $\varepsilon^{\prime \prime}$ according to eq. (4.31). Let us introduce the set of indices

$$
I^{\prime}=\left\{1,2, \ldots, i_{0}, e\right\}, \quad I^{\prime \prime}=\left\{e, i_{0}+1, i_{0}+2, \ldots, n\right\} .
$$

We denote by $S_{i_{0}-1}^{(1, e)}$ the set of permutations $\kappa^{\prime}$ of the set $I^{\prime}$, where the values $\kappa_{1}^{\prime}=1$ and $\kappa_{i_{0}+1}^{\prime}=e$ are fixed. Similarly, we denote by $S_{n-i_{0}-1}^{(e, n)}$ the set of permutations $\kappa^{\prime \prime}$ of the set $I^{\prime \prime}$, where the values $\kappa_{1}^{\prime \prime}=e$ and $\kappa_{n-i_{0}+1}^{\prime \prime}=n$ are fixed. For

$$
\kappa^{\prime}=\left(1, \kappa_{2}^{\prime}, \ldots, \kappa_{i_{0}}^{\prime}, e\right) \in S_{i_{0}-1}^{(1, e)}, \quad \kappa^{\prime \prime}=\left(e, \kappa_{i_{0}+1}^{\prime \prime}, \ldots, \kappa_{n-1}^{\prime \prime}, n\right) \in S_{n-i_{0}-1}^{(e, n)}
$$

we define $\kappa=\left(\kappa^{\prime}, \kappa^{\prime \prime}\right)$ by

$$
\kappa=\left(1, \kappa_{2}^{\prime}, \ldots, \kappa_{i_{0}}^{\prime}, \kappa_{i_{0}+1}^{\prime \prime}, \ldots, \kappa_{n-1}^{\prime \prime}, n\right) .
$$

We would like to show

$$
\operatorname{Res}_{Y} \Omega_{\mathrm{scattering}}^{\mathrm{pol}}(p, \varepsilon, z)=\sum_{f, \lambda}(-1)^{i_{0}-1} \Omega_{\mathrm{scattering}}^{\mathrm{pol}}\left(p^{\prime}, \varepsilon^{\prime}, z\right) \wedge \Omega_{\mathrm{scattering}}^{\mathrm{pol}}\left(p^{\prime \prime}, \varepsilon^{\prime \prime}, z\right) .
$$


Let us first look at the left-hand side. From the definition in eq. (4.41) we have

$$
\operatorname{Res}_{Y} \Omega_{\text {scattering }}^{\text {pol }}(p, \varepsilon, z)=\sum_{\kappa \in S_{n-2}^{(1, n)}} N_{\text {comb }}^{\mathrm{BCJ}}(\kappa) \operatorname{Res}_{Y} \Omega_{\text {cyclic }}^{\text {pol }}(\kappa, z) .
$$

From section 4.1 we know that there is only a residue if $\kappa \sim_{\left(i_{0}, n\right)} \pi$. This is the case, if $\kappa$ can be written as $\kappa=\left(\kappa^{\prime}, \kappa^{\prime \prime}\right)$ with $\kappa^{\prime} \in S_{i_{0}-1}^{(1, e)}$ and $\kappa^{\prime \prime} \in S_{n-i_{0}-1}^{(e, n)}$. Therefore

$$
\text { 1.h.s. }=(-1)^{i_{0}-1} \sum_{\kappa^{\prime} \in S_{i_{0}-1}^{(1, e)}} \sum_{\kappa^{\prime \prime} \in S_{n-i_{0}-1}^{(e, n)}} N_{\text {comb }}^{\mathrm{BCJ}}\left(\left(\kappa^{\prime}, \kappa^{\prime \prime}\right)\right) \Omega_{\text {scattering }}^{\text {cyclic }}\left(\kappa^{\prime}, z\right) \wedge \Omega_{\text {scattering }}^{\text {cyclic }}\left(\kappa^{\prime \prime}, z\right) \text {. }
$$

Now, $N_{\text {comb }}^{\mathrm{BCJ}}\left(\left(\kappa^{\prime}, \kappa^{\prime \prime}\right)\right)$ is the BCJ-numerator of a multi-peripheral graph and factorises as

$$
N_{\mathrm{comb}}^{\mathrm{BCJ}}\left(\left(\kappa^{\prime}, \kappa^{\prime \prime}\right)\right)=\sum_{f, \lambda} N_{\mathrm{comb}}^{\mathrm{BCJ}}\left(\kappa^{\prime}\right) N_{\mathrm{comb}}^{\mathrm{BCJ}}\left(\kappa^{\prime \prime}\right) .
$$

Therefore

l.h.s. $=$

$$
(-1)^{i_{0}-1} \sum_{f, \lambda} \sum_{\kappa^{\prime} \in S_{i_{0}-1}^{(1, e)}} N_{\mathrm{comb}}^{\mathrm{BCJ}}\left(\kappa^{\prime}\right) \Omega_{\mathrm{scattering}}^{\text {cyclic }}\left(\kappa^{\prime}, z\right) \wedge \sum_{\kappa^{\prime \prime} \in S_{n-i_{0}-1}^{(e, n)}} N_{\text {comb }}^{\mathrm{BCJ}}\left(\kappa^{\prime \prime}\right) \Omega_{\text {scattering }}^{\text {cyclic }}\left(\kappa^{\prime \prime}, z\right),
$$

and hence

$$
\text { l.h.s. }=\sum_{f, \lambda}(-1)^{i_{0}-1} \Omega_{\mathrm{scattering}}^{\mathrm{pol}}\left(p^{\prime}, \varepsilon^{\prime}, z\right) \wedge \Omega_{\mathrm{scattering}}^{\mathrm{pol}}\left(p^{\prime \prime}, \varepsilon^{\prime \prime}, z\right),
$$

which proves the claim.

\subsubsection{Examples}

Let us look at a few examples. We consider again $\mathcal{M}_{0, n}^{\pi}$ with $\pi=(1,2, \ldots, n)$. We fix $z_{1}=0, z_{n-1}=1$ and $z_{n}=\infty$. We look at the scattering form $\Omega_{\text {scattering }}^{\text {pol }}$ for external gauge bosons. The simplest case is $n=3$. We have the 0 -form

$$
\begin{aligned}
& \Omega_{\text {scattering }}^{\text {pol }}(p, \varepsilon, z)= \\
& \quad\left(\varepsilon_{1} \cdot \varepsilon_{2}\right)\left(\varepsilon_{3} \cdot\left(p_{1}-p_{2}\right)\right)+\left(\varepsilon_{2} \cdot \varepsilon_{3}\right)\left(\varepsilon_{1} \cdot\left(p_{2}-p_{3}\right)\right)+\left(\varepsilon_{3} \cdot \varepsilon_{1}\right)\left(\varepsilon_{2} \cdot\left(p_{3}-p_{1}\right)\right) .
\end{aligned}
$$

$\mathrm{Up}$ to a factor $i$, this is the three-point amplitude or equivalently the three-point vertex.

The next case is $n=4$. Here we have

$$
\Omega_{\mathrm{scattering}}^{\mathrm{pol}}(p, \varepsilon, z)=\left[\frac{N_{\mathrm{comb}}^{\mathrm{BCJ}}((1,2,3,4))}{u_{2,4}\left(u_{2,4}-1\right)}-\frac{N_{\mathrm{comb}}^{\mathrm{BCJ}}((1,3,2,4))}{\left(u_{2,4}-1\right)}\right] d u_{2,4},
$$

with

$$
\begin{aligned}
& N_{\mathrm{comb}}^{\mathrm{BCJ}}((1,2,3,4))= \\
& \quad 4\left(p_{1} \cdot \varepsilon_{2}\right)\left(p_{4} \cdot \varepsilon_{3}\right)\left(\varepsilon_{1} \cdot \varepsilon_{4}\right)-4\left(p_{1} \cdot \varepsilon_{2}\right)\left(p_{3} \cdot \varepsilon_{4}\right)\left(\varepsilon_{1} \cdot \varepsilon_{3}\right)-4\left(p_{2} \cdot \varepsilon_{1}\right)\left(p_{4} \cdot \varepsilon_{3}\right)\left(\varepsilon_{2} \cdot \varepsilon_{4}\right) \\
& +4\left(p_{2} \cdot \varepsilon_{1}\right)\left(p_{3} \cdot \varepsilon_{4}\right)\left(\varepsilon_{2} \cdot \varepsilon_{3}\right)-4\left[\left(p_{1} \cdot \varepsilon_{3}\right)\left(p_{2} \cdot \varepsilon_{4}\right)-\left(p_{1} \cdot \varepsilon_{4}\right)\left(p_{2} \cdot \varepsilon_{3}\right)\right]\left(\varepsilon_{1} \cdot \varepsilon_{2}\right) \\
& -4\left[\left(p_{3} \cdot \varepsilon_{1}\right)\left(p_{4} \cdot \varepsilon_{2}\right)-\left(p_{3} \cdot \varepsilon_{2}\right)\left(p_{4} \cdot \varepsilon_{1}\right)\right]\left(\varepsilon_{3} \cdot \varepsilon_{4}\right)-2\left(p_{1} \cdot p_{2}\right)\left(\varepsilon_{1} \cdot \varepsilon_{3}\right)\left(\varepsilon_{2} \cdot \varepsilon_{4}\right) \\
& +2\left(p_{1} \cdot p_{2}\right)\left(\varepsilon_{1} \cdot \varepsilon_{4}\right)\left(\varepsilon_{2} \cdot \varepsilon_{3}\right)+2\left(p_{2} \cdot p_{3}-p_{1} \cdot p_{3}\right)\left(\varepsilon_{1} \cdot \varepsilon_{2}\right)\left(\varepsilon_{3} \cdot \varepsilon_{4}\right),
\end{aligned}
$$

and $N_{\text {comb }}^{\mathrm{BCJ}}((1,3,2,4))$ is obtained from $N_{\text {comb }}^{\mathrm{BCJ}}((1,2,3,4))$ by $2 \leftrightarrow 3$. 
Finally, let us consider the case $n=5$. Here we have

$$
\begin{aligned}
& \Omega_{\text {scattering }}^{\text {pol }}(p, \varepsilon, z)=\left[\frac{N_{\text {comb }}^{\mathrm{BCJ}}((1,2,3,4,5))}{u_{2,5}\left(u_{2,5}-1\right) u_{3,5}\left(u_{3,5}-1\right)}-\frac{N_{\text {comb }}^{\mathrm{BCJ}}((1,3,4,2,5))}{\left(u_{3,5}-1\right)\left(u_{2,5} u_{3,5}-1\right)}\right. \\
& -\frac{N_{\text {comb }}^{\mathrm{BCJ}}((1,4,2,3,5))}{\left(u_{2,5}-1\right)\left(u_{2,5} u_{3,5}-1\right)}+\frac{N_{\mathrm{comb}}^{\mathrm{BCJ}}((1,4,3,2,5))}{\left(u_{2,5}-1\right)\left(u_{3,5}-1\right)}-\frac{N_{\mathrm{comb}}^{\mathrm{BCJ}}((1,2,4,3,5))}{u_{2,5}\left(u_{3,5}-1\right)\left(u_{2,5} u_{3,5}-1\right)} \\
& \left.-\frac{N_{\mathrm{comb}}^{\mathrm{BCJ}}((1,3,2,4,5))}{\left(u_{2,5}-1\right) u_{3,5}\left(u_{2,5} u_{3,5}-1\right)}\right] d u_{2,5} \wedge d u_{3,5} .
\end{aligned}
$$

We may rewrite this expression with the help of the Leinartas decomposition [42-44] as

$$
\begin{aligned}
& \Omega_{\text {scattering }}^{\mathrm{pol}}(p, \varepsilon, z)=\left[\frac{N_{\mathrm{comb}}^{\mathrm{BCJ}}((1,2,3,4,5))}{u_{2,5} u_{3,5}}+\frac{N_{\mathrm{comb}}^{\mathrm{BCJ}}((1,2,4,3,5))-N_{\mathrm{comb}}^{\mathrm{BCJ}}((1,2,3,4,5))}{u_{2,5}\left(u_{3,5}-1\right)}\right. \\
& +\frac{N_{\mathrm{comb}}^{\mathrm{BCJ}}((1,3,2,4,5))-N_{\mathrm{comb}}^{\mathrm{BCJ}}((1,2,3,4,5))}{\left(u_{2,5}-1\right) u_{3,5}}+\frac{N_{\mathrm{comb}}^{\mathrm{BCJ}}((1,4,3,2,5))+N_{\mathrm{comb}}^{\mathrm{BCJ}}((1,2,3,4,5))}{\left(u_{2,5}-1\right)\left(u_{3,5}-1\right)} \\
& -\frac{N_{\mathrm{comb}}^{\mathrm{BCJ}}((1,4,2,3,5))+N_{\mathrm{comb}}^{\mathrm{BCJ}}((1,3,2,4,5))}{\left(u_{2,5}-1\right)\left(u_{2,5} u_{3,5}-1\right)}-\frac{N_{\mathrm{comb}}^{\mathrm{BCJ}}((1,3,4,2,5))+N_{\mathrm{comb}}^{\mathrm{BCJ}}((1,2,4,3,5))}{\left(u_{3,5}-1\right)\left(u_{2,5} u_{3,5}-1\right)} \\
& \left.-\frac{N_{\mathrm{comb}}^{\mathrm{BCJ}}((1,2,4,3,5))+N_{\mathrm{comb}}^{\mathrm{BCJ}}((1,3,2,4,5))}{\left(u_{2,5} u_{3,5}-1\right)}\right] d u_{2,5} \wedge d u_{3,5} .
\end{aligned}
$$

All occuring BCJ-numerators may be obtained from the BCJ-numerator $N_{\text {comb }}^{\text {BCJ }}((1,2,3,4,5))$ by a suitable substitution of the indices. The explicit expression for $N_{\mathrm{comb}}^{\mathrm{BCJ}}((1,2,3,4,5))$ is rather long and not reproduced here. It is obtained in a straightforward way from the effective Lagrangian in eq. (2.21). Let us however point out that $N_{\text {comb }}^{\mathrm{BCJ}}((1,2,3,4,5))$ is not unique. This is related to the free parameter $a$ in eq. (2.25).

\subsection{Summary on the factorisation of the residues}

At the end of this section, let us summarise the factorisation properties of the residues of the scattering forms $\Omega_{\text {scattering }}^{\text {cyclic }}(\sigma, z)$ and $\Omega_{\text {scattering }}^{\text {pol }}(p, \varepsilon, z)$. On $\mathcal{M}_{0, n}^{\pi}$ with $\pi=(1,2, \ldots, n)$ we have in the limit $u_{i_{0}, n} \rightarrow 0$

$$
\begin{aligned}
& \operatorname{Res}_{Y} \Omega_{\text {scattering }}^{\text {cyclic }}(\sigma, z)= \begin{cases}(-1)^{i_{0}-1} \Omega_{\text {scattering }}^{\text {cyclic }}\left(\sigma^{\prime}, z\right) \wedge \Omega_{\text {scattering }}^{\text {cyclic }}\left(\sigma^{\prime \prime}, z\right) & \text { if } \sigma \sim_{\left(i_{0}, n\right)} \pi, \\
0 & \text { otherwise. }\end{cases} \\
& \operatorname{Res}_{Y} \Omega_{\mathrm{scattering}}^{\mathrm{pol}}(p, \varepsilon, z)=(-1)^{i_{0}-1} \sum_{f, \lambda} \Omega_{\mathrm{scattering}}^{\mathrm{pol}}\left(p^{\prime}, \varepsilon^{\prime}, z\right) \wedge \Omega_{\mathrm{scattering}}^{\mathrm{pol}}\left(p^{\prime \prime}, \varepsilon^{\prime \prime}, z\right),
\end{aligned}
$$

where $Y$ denotes the hypersurface $u_{i_{0}, n}=0$.

\section{Conclusions}

In this paper we studied the properties of the scattering forms $\Omega_{\text {scattering }}^{\text {cyclic }}$ and $\Omega_{\text {scattering }}^{\text {pol }}$. These are two differential $(n-3)$-forms defined on the compactifiaction $\overline{\mathcal{M}}_{0, n}$ of the moduli space of a Riemann surface of genus 0 with $n$ marked points. The scattering forms are cocycles. The scattering equations define a one-form $\eta$ and Mizera [5] has shown recently 
that the scattering amplitudes are given as intersection numbers of the scattering forms twisted by the one-form $\eta$. With the two scattering forms $\Omega_{\text {scattering }}^{\text {cyclic }}$ and $\Omega_{\text {scattering }}^{\text {pol }}$ at hand we obtain, depending on the combination we take, the scattering amplitudes within the bi-adjoint scalar theory, Yang-Mills theory and gravity.

In this paper we investigated the scattering forms in more detail. We studied them on the complete $(n-3)$-dimensional space $\overline{\mathcal{M}}_{0, n}$, not just on the zero-dimensional sub-variety defined by the scattering equations.

The scattering forms have some remarkable properties, given at the beginning of section 4. We have shown that the only singularities of the scattering forms are on the divisor $\overline{\mathcal{M}}_{0, n} \backslash \mathcal{M}_{0, n}$, that all singularities are logarithmic and that the residues at the singularities factorise into two scattering forms of lower points. These properties provide a direct bridge from the scattering forms, obtained from the CHY representation, to recent ideas involving associahedra and amplituhedra [4, 45-50]. It is probably fair to say that we now have a clear geometric picture of tree-level amplitudes within the bi-adjoint scalar theory, Yang-Mills theory and gravity for any number of external particles $n$.

We expect these ideas to be fruitful for a wider set of theories [51-63]. Another promising and interesting direction is to explore these ideas beyond tree-level amplitudes.

\section{Acknowledgments}

A.K. is grateful for financial support from the research training group GRK 1581.

\section{A The reduced Pfaffian}

\section{A.1 Definition}

In this appendix we collect the original definition of the polarisation factor $E(p, \varepsilon, z)$ in terms of a reduced Pfaffian. We start from a $(2 n) \times(2 n)$ anti-symmetric matrix $\Psi$

$$
\Psi=\left(\begin{array}{cc}
A & -C^{T} \\
C & B
\end{array}\right)
$$

with

$$
A_{a b}=\left\{\begin{array}{cc}
\frac{2 p_{a} \cdot p_{b}}{z_{a}-z_{b}} & a \neq b, \\
0 & a=b,
\end{array} B_{a b}=\left\{\begin{array}{cc}
\frac{2 \varepsilon_{a} \cdot \varepsilon_{b}}{z_{a}-z_{b}} & a \neq b \\
0 & a=b
\end{array}\right.\right.
$$

and

$$
C_{a b}=\left\{\begin{array}{cr}
\frac{2 \varepsilon_{a} \cdot p_{b}}{z_{a}-z_{b}} \quad a \neq b, \\
-\sum_{j=1, j \neq a}^{n} \frac{2 \varepsilon_{a} \cdot p_{j}}{z_{a}-z_{j}} a=b .
\end{array}\right.
$$

Let $1 \leq i<j \leq n$. One denotes by $\Psi_{i j}^{i j}$ the $(2 n-2) \times(2 n-2)$-matrix where the rows and columns $i$ and $j$ of $\Psi$ have been deleted. $\Psi_{i j}^{i j}$ has a non-vanishing Pfaffian. The original definition of the polarisation factor reads $[1-3]$

$$
E(p, \varepsilon, z)=\frac{(-1)^{i+j}}{2\left(z_{i}-z_{j}\right)} \operatorname{Pf} \Psi_{i j}^{i j} .
$$


This expression is independent of the choice of $i$ and $j$ if the momenta $p$ are on-shell, the polarisation vectors $\varepsilon$ are transverse and the variables $z$ are solutions of the scattering equations.

\section{A.2 A counter-example}

In this appendix we show that the reduced Pfaffian is not suited to define the polarisation scattering form. Let us start from

$$
\frac{(-1)^{i+j} \operatorname{Pf} \Psi_{i j}^{i j}}{2\left(z_{i}-z_{j}\right)} \frac{d^{n} z}{d \omega} .
$$

The first problem we face is that this expression is not independent of the deleted rows and columns $i$ and $j$ as soon as we go away from the solutions of the scattering equations. We may overcome this problem by averaging over all possible choices of $i$ and $j$. Thus we are tempted to consider

$$
\Omega^{\text {try }}(p, \varepsilon, z)=\frac{2}{n(n-1)} \sum_{i<j} \frac{(-1)^{i+j} \operatorname{Pf} \Psi_{i j}^{i j}}{2\left(z_{i}-z_{j}\right)} \frac{d^{n} z}{d \omega} .
$$

Let us specialise to the case $n=4$. One may show that $\Omega^{\text {try }}$ is $\operatorname{PSL}(2, \mathbb{C})$-invariant. We are considering $\mathcal{M}_{0,4}^{\pi}$ with $\pi=(1,2,3,4)$. With the gauge choice $z_{1}=0, z_{3}=1$ and $z_{4}=\infty$ one finds

$$
\Omega^{\operatorname{try}}(p, \varepsilon, z)=-\frac{2}{3}\left(\varepsilon_{1} \cdot \varepsilon_{2}\right)\left(\varepsilon_{3} \cdot \varepsilon_{4}\right) s_{12} \frac{d u_{2,4}}{u_{2,4}^{2}}+\ldots,
$$

where the dots denote terms less singular at $u_{2,4}=0$. Thus $\Omega^{\text {try }}$ has higher poles and does not satisfy the property of having only logarithmic singularities on the divisor $\overline{\mathcal{M}}_{0, n} \backslash \mathcal{M}_{0, n}$.

\section{B Reduction of the numerators}

Let $\sigma=(1,2, \ldots, n)$ be a cyclic order and $G \in \mathcal{T}_{n}(\sigma)$ a graph with this cyclic order. In this appendix we show that

$$
N^{\mathrm{BCJ}}(G)=\hat{N}^{\mathrm{BCJ}}(G),
$$

where $\hat{N}^{\mathrm{BCJ}}(G)$ is defined by

$$
\hat{N}^{\mathrm{BCJ}}(G)=\sum_{\tilde{\sigma} \in \mathrm{CO}(G)} \sum_{\kappa \in S_{n-2}^{(1, n)}} \delta_{\tilde{\sigma}, \kappa}(-1)^{n_{\mathrm{flip}}(\sigma, \kappa)} N_{\mathrm{comb}}^{\mathrm{BCJ}}(\kappa) .
$$

Eq. (B.1) gives the reduction of an arbitrary BCJ-numerator into the basis of multiperipheral BCJ-numerators by repeated use of eq. (2.26). Let us first look at an example. Figure 7 shows an example of a generic graph with the cyclic order $(1,2, \ldots, 10)$. If we apply the STU-relation at the vertex $v_{1}$, we obtain the two graphs shown in figure 8 . The left graph has the cyclic order $(1,2,3,4,5,6,7,8,9,10)$ and comes with a plus sign, the right graph has the cyclic order $(1,2,5,6,3,4,7,8,9,10)$ and comes with a minus sign. These are exactly the cyclic orders (with the correct sign) we obtain from swapping the branches 


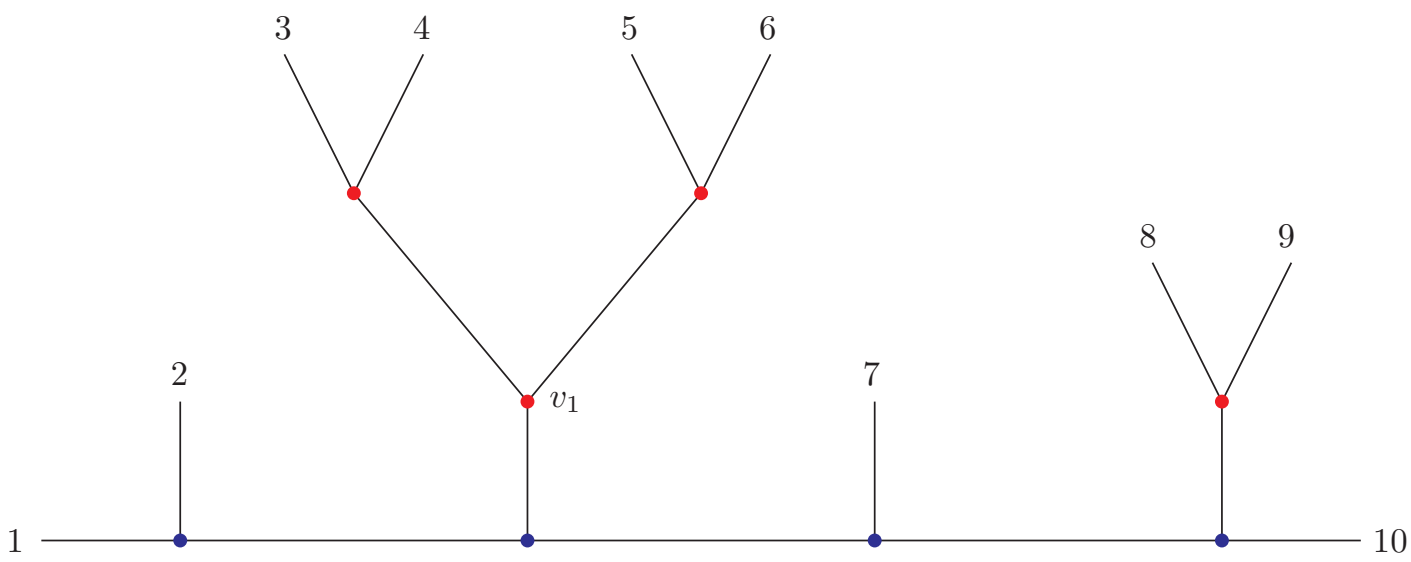

Figure 7. An example of a generic graph with the cyclic order $(1,2, \ldots, 10)$. Vertices on the line connecting particle 1 with 10 are drawn in blue, all other vertices are drawn in red.
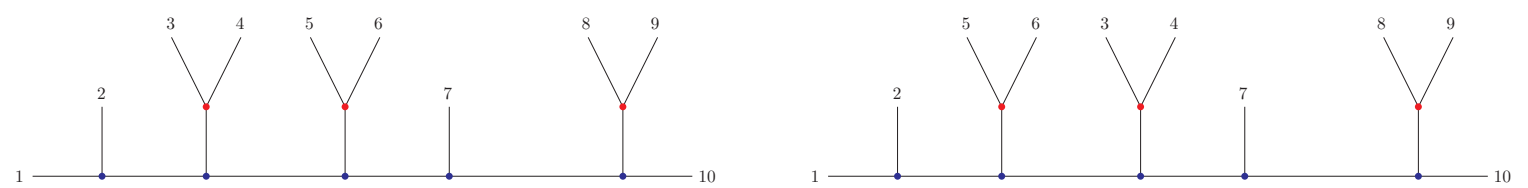

Figure 8. Reduction of the graph from figure 7: a single application of the STU-relation at the vertex $v_{1}$ yields the two graphs shown in this figure. The left graph occurs with a plus sign, the right graph with a minus sign.

at the vertex $v_{1}$. We have a one-to-one correspondence between the terms occurring in the decomposition of $N^{\mathrm{BCJ}}(G)$ into a multi-peripheral basis and the cyclic orders obtained by swapping in all possible ways the vertices indicated by red (but not the ones shown in blue) in figure 7 . Let us adopt the convention, that in any cyclic order leg 10 occurs in the last place. Then, swapping the branches at a red vertex will keep leg 1 in the first place, whereas swapping the branches at a blue vertex will give a cyclic order where leg 1 is not in the first place. We may therefore sum over all cyclic orders compatible with $G$ and veto the ones which correspond to a swap at a blue vertex. The ones which correspond to swaps at red vertices only are the cyclic orders $\kappa \in S_{n-2}^{(1, n)}$. We thus arrive at

$$
N^{\mathrm{BCJ}}(G)=\sum_{\tilde{\sigma} \in \mathrm{CO}(G)} \sum_{\kappa \in S_{n-2}^{(1, n)}} \delta_{\tilde{\sigma}, \kappa}(-1)^{n_{\text {flip }}(\sigma, \kappa)} N_{\mathrm{comb}}^{\mathrm{BCJ}}(\kappa),
$$

where $\delta_{\tilde{\sigma}, \kappa}$ selects exactly the cyclic orders, which are obtained from swapping branches at the red vertices only.

\section{Permutation invariance of the polarisation factor}

In this appendix we show the permutation invariance of the polarisation factor. We set

$$
E_{1, n}=\sum_{\kappa \in S_{n-2}^{(1, n)}} C(\kappa, z) N_{\mathrm{comb}}^{\mathrm{BCJ}}(\kappa),
$$






Figure 9. The graph $F^{\prime}$ obtained from the graph underlying the numerator $N_{\text {comb }}^{\mathrm{BCJ}}\left(\left(1, \alpha^{\prime}, n, \beta^{\prime}, j\right)\right)$ by swapping the branches at the vertex where leg $n$ is attached.

$$
E_{i, j}=\sum_{\kappa \in S_{n-2}^{(i, j)}} C(\kappa, z) N_{\mathrm{comb}}^{\mathrm{BCJ}}(\kappa)
$$

We would like to show that

$$
E_{i, j}=E_{1, n}
$$

We may split this into two steps, by first establishing $E_{i, j}=E_{1, j}$ and then in a second step $E_{1, j}=E_{1, n}$. The proof for the step, which exchanges $i \leftrightarrow 1$ is similar to the proof for the step, which exchanges $j \leftrightarrow n$, therefore it suffices to discuss one case. We show

$$
E_{1, j}=E_{1, n}
$$

$E_{1, j}$ is given by

$$
E_{1, j}=\sum_{\kappa^{\prime} \in S_{n-2}^{(1, j)}} C\left(\kappa^{\prime}, z\right) N_{\mathrm{comb}}^{\mathrm{BCJ}}\left(\kappa^{\prime}\right)
$$

where $\kappa^{\prime}$ is of the form $\left(1, \alpha^{\prime}, n, \beta^{\prime}, j\right)$ with $\alpha^{\prime} \cup \beta^{\prime}=\{2, \ldots, n-1\} \backslash\{j\}$ and $\alpha^{\prime} \cap \beta^{\prime}=\emptyset$. We may replace the sum over $\kappa^{\prime}$ by a sum over all possible choices for $\alpha^{\prime}$ and $\beta^{\prime}$. Thus we write

$$
E_{1, j}=\sum_{\alpha^{\prime}, \beta^{\prime}} C\left(\left(1, \alpha^{\prime}, n, \beta^{\prime}, j\right), z\right) N_{\mathrm{comb}}^{\mathrm{BCJ}}\left(\left(1, \alpha^{\prime}, n, \beta^{\prime}, j\right)\right) .
$$

Let $G^{\prime}$ be the multi-peripheral graph corresponding to the BCJ-numerator $N_{\text {comb }}^{\text {BCJ }}\left(\left(1, \alpha^{\prime}, n, \beta^{\prime}, j\right)\right)$. We denote by $F^{\prime}$ the graph obtained from the graph $G^{\prime}$ by swapping the branches at the vertex where leg $n$ is attached. The graph $F^{\prime}$ has the cyclic order $\kappa_{F}^{\prime}=\left(1, \alpha^{\prime}, \beta^{\prime}, j, n\right)$. An example is shown in figure 9 . We have

$$
N^{\mathrm{BCJ}}\left(G^{\prime}\right)=-N^{\mathrm{BCJ}}\left(F^{\prime}\right) .
$$

Clearly

$$
\mathrm{CO}\left(G^{\prime}\right)=\mathrm{CO}\left(F^{\prime}\right)
$$

and for any cyclic order $\sigma$

$$
(-1)^{n_{\mathrm{flip}}\left(\kappa^{\prime}, \sigma\right)}=-(-1)^{n_{\mathrm{flip}}\left(\kappa_{F}^{\prime}, \sigma\right)}
$$

since $F^{\prime}$ differs from $G^{\prime}$ by exactly one swap. From eq. (B.3) it follows that

$$
N^{\mathrm{BCJ}}\left(G^{\prime}\right)=\sum_{\tilde{\sigma} \in \mathrm{CO}\left(G^{\prime}\right)} \sum_{\kappa \in S_{n-2}^{(1, n)}} \delta_{\tilde{\sigma}, \kappa}(-1)^{n_{\mathrm{flip}}\left(\kappa^{\prime}, \kappa\right)} N_{\mathrm{comb}}^{\mathrm{BCJ}}(\kappa) .
$$


Thus

$$
E_{1, j}=\sum_{\kappa \in S_{n-2}^{(1, n)}} N_{\mathrm{comb}}^{\mathrm{BCJ}}(\kappa) \sum_{\kappa^{\prime} \in S_{n-2}^{(1, j)}} C\left(\kappa^{\prime}, z\right) \sum_{\tilde{\sigma} \in \mathrm{CO}\left(G^{\prime}\right)} \delta_{\tilde{\sigma}, \kappa}(-1)^{n_{\text {flip }}\left(\kappa^{\prime}, \kappa\right)} .
$$

This equals $E_{1, n}$ if

$$
C(\kappa, z)=\sum_{\alpha^{\prime}, \beta^{\prime}} C\left(\kappa^{\prime}, z\right) \sum_{\tilde{\sigma} \in \operatorname{CO}\left(G^{\prime}\right)} \delta_{\tilde{\sigma}, \kappa}(-1)^{n_{\text {flip }}\left(\kappa^{\prime}, \kappa\right)},
$$

with $\kappa^{\prime}=\left(1, \alpha^{\prime}, n, \beta^{\prime}, j\right)$. With the help of the Kleiss-Kuijf relations we may express $C\left(\kappa^{\prime}, z\right)$ as

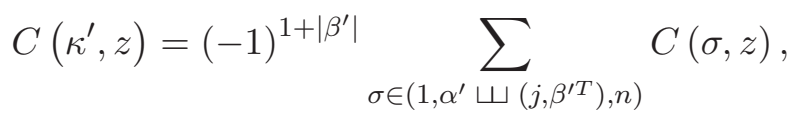

where $\left|\beta^{\prime}\right|$ denotes the number of elements of $\beta^{\prime}$. Thus the right-hand side of eq. (C.11) equals

$$
\begin{aligned}
\text { r.h.s. } & =\sum_{\alpha^{\prime}, \beta^{\prime}}(-1)^{1+\left|\beta^{\prime}\right|} \sum_{\sigma \in\left(1, \alpha^{\prime} \amalg\left(j, \beta^{\prime T}\right), n\right)} C(\sigma, z) \sum_{\tilde{\sigma} \in \mathrm{CO}\left(G^{\prime}\right)} \delta_{\tilde{\sigma}, \kappa}(-1)^{n_{\text {flip }}\left(\kappa^{\prime}, \kappa\right)} \\
& =\sum_{\kappa^{\prime \prime} \in S_{n-2}^{(1, n)}} C\left(\kappa^{\prime \prime}, z\right) \sum_{\alpha^{\prime}, \beta^{\prime}}(-1)^{1+\left|\beta^{\prime}\right|} \sum_{\sigma \in\left(1, \alpha^{\prime}\right.} \sum_{\left.\left(j, \beta^{\prime T}\right), n\right)} \delta_{\kappa^{\prime \prime}, \sigma} \sum_{\tilde{\sigma} \in \operatorname{CO}\left(G^{\prime}\right)} \delta_{\tilde{\sigma}, \kappa}(-1)^{n_{\text {flip }}\left(\kappa^{\prime}, \kappa\right)} .
\end{aligned}
$$

Therefore it remains to show

$$
\delta_{\kappa, \kappa^{\prime \prime}}=\sum_{\kappa^{\prime}=\left(1, \alpha^{\prime}, n, \beta^{\prime}, j\right) \in S_{n-2}^{(1, j)}} \sum_{\sigma \in\left(1, \alpha^{\prime}\right.} \sum_{\left.\left(j, \beta^{\prime T}\right), n\right)} \sum_{\tilde{\sigma} \in \mathrm{CO}\left(G^{\prime}\right)} \delta_{\kappa^{\prime \prime}, \sigma} \delta_{\tilde{\sigma}, \kappa}(-1)^{1+\left|\beta^{\prime}\right|+n_{\mathrm{flip}}\left(\kappa^{\prime}, \kappa\right)} .
$$

Let us first discuss the case $\kappa=\kappa^{\prime \prime}$. Without loss of generality we may assume $\kappa=\kappa^{\prime \prime}=$ $(1,2, \ldots, n)$. The right-hand side of eq. (C.14) simplifies to

$$
\text { r.h.s. }=\sum_{\kappa^{\prime}=\left(1, \alpha^{\prime}, n, \beta^{\prime}, j\right) \in S_{n-2}^{(1, j)}} \sum_{\sigma \in\left(1, \alpha^{\prime} \amalg\left(j, \beta^{\prime T}\right), n\right)} \sum_{\tilde{\sigma} \in \mathrm{CO}\left(G^{\prime}\right)} \delta_{\kappa, \sigma} \delta_{\sigma, \tilde{\sigma}}(-1)^{1+\left|\beta^{\prime}\right|+n_{\mathrm{flip}}\left(\kappa^{\prime}, \kappa\right)} .
$$

The Kronecker delta $\delta_{\sigma, \tilde{\sigma}}$ selects from the sums over $\sigma$ and $\tilde{\sigma}$ the term $\sigma=\tilde{\sigma}=$ $\left(1, \alpha^{\prime}, j, \beta^{T}, n\right)$. In addition we must have $\kappa=\sigma$, i.e. $\alpha^{\prime}=(2, \ldots, j-1)$ and $\beta^{\prime T}=(j+1, \ldots, n-1)$. Therefore also the sum over $\kappa^{\prime}$ reduces to one term. For $\kappa^{\prime}=(1,2, \ldots, j-1, n, n-1, \ldots, j+1, j)$ we have

$$
n_{\text {flip }}\left(\kappa^{\prime}, \kappa\right)=n-j .
$$

We obtain for the right-hand side of eq. (C.14)

$$
\text { r.h.s. }=(-1)^{1+\left|\beta^{\prime}\right|+n_{\text {flip }}\left(\kappa^{\prime}, \kappa\right)}=(-1)^{1+(n-1-j)+(n-j)}=1 .
$$

Let us now discuss for $\kappa, \kappa^{\prime \prime} \in S_{n-2}^{(1, n)}$ the case $\kappa \neq \kappa^{\prime \prime}$. This requires $n \geq 4$. Without loss of generality we may take $\kappa^{\prime \prime}=(1,2, \ldots, n)$ and $j=n-1$. The Kronecker delta $\delta_{\kappa^{\prime \prime}, \sigma}$ 
enforces then $\beta^{\prime}=\emptyset$ and $\alpha^{\prime}=(2, \ldots, n-2)$. We thus have $\kappa^{\prime}=(1,2, \ldots, n-2, n, n-1)$. The graph $G^{\prime}$ is then the multi-peripheral graph

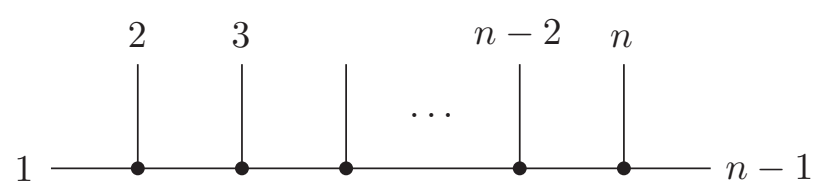

and the graph $F^{\prime}$ is the multi-peripheral graph

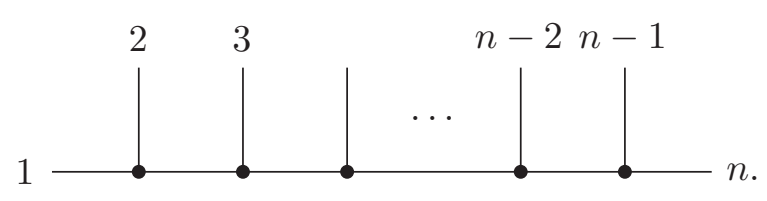

The only cyclic order of $\mathrm{CO}\left(G^{\prime}\right)$, which is also an element of $S_{n-2}^{(1, n)}$ is

$$
(1,2, \ldots, n)
$$

Thus, the Kronecker delta $\delta_{\tilde{\sigma}, \kappa}$ enforces $\kappa=\tilde{\sigma}=(1,2, \ldots, n)$. Thus $\kappa=\kappa^{\prime \prime}$. But this is a contradiction to the assumption $\kappa \neq \kappa^{\prime \prime}$. Therefore all terms on the right-hand side of eq. (C.14) vanish due to the Kronecker deltas and the right-hand side of eq. (C.14) yields zero for $\kappa \neq \kappa^{\prime \prime}$. This completes the proof of the permutation invariance of the polarisation factor.

Open Access. This article is distributed under the terms of the Creative Commons Attribution License (CC-BY 4.0), which permits any use, distribution and reproduction in any medium, provided the original author(s) and source are credited.

\section{References}

[1] F. Cachazo, S. He and E.Y. Yuan, Scattering equations and Kawai-Lewellen-Tye orthogonality, Phys. Rev. D 90 (2014) 065001 [arXiv:1306.6575] [INSPIRE].

[2] F. Cachazo, S. He and E.Y. Yuan, Scattering of Massless Particles in Arbitrary Dimensions, Phys. Rev. Lett. 113 (2014) 171601 [arXiv:1307.2199] [INSPIRE].

[3] F. Cachazo, S. He and E.Y. Yuan, Scattering of Massless Particles: Scalars, Gluons and Gravitons, JHEP 07 (2014) 033 [arXiv:1309.0885] [InSPIRE].

[4] N. Arkani-Hamed, Y. Bai and T. Lam, Positive Geometries and Canonical Forms, JHEP 11 (2017) 039 [arXiv:1703.04541] [INSPIRE].

[5] S. Mizera, Scattering Amplitudes from Intersection Theory, arXiv:1711.00469 [INSPIRE].

[6] S. Mizera, Combinatorics and Topology of Kawai-Lewellen-Tye Relations, JHEP 08 (2017) 097 [arXiv: 1706.08527] [INSPIRE].

[7] F. Cachazo, S. Mizera and G. Zhang, Scattering Equations: Real Solutions and Particles on a Line, JHEP 03 (2017) 151 [arXiv:1609.00008] [INSPIRE]. 
[8] S. Weinzierl, On the solutions of the scattering equations, JHEP 04 (2014) 092 [arXiv: 1402.2516] [INSPIRE].

[9] C. Kalousios, Massless scattering at special kinematics as Jacobi polynomials, J. Phys. A 47 (2014) 215402 [arXiv:1312.7743] [InSPIRE].

[10] N. Arkani-Hamed, Y. Bai, S. He and G. Yan, Scattering Forms and the Positive Geometry of Kinematics, Color and the Worldsheet, arXiv:1711.09102 [INSPIRE].

[11] M. Tolotti and S. Weinzierl, Construction of an effective Yang-Mills Lagrangian with manifest BCJ duality, JHEP 07 (2013) 111 [arXiv:1306.2975] [INSPIRE].

[12] P. Draggiotis, R.H.P. Kleiss and C.G. Papadopoulos, On the computation of multigluon amplitudes, Phys. Lett. B 439 (1998) 157 [hep-ph/9807207] [INSPIRE].

[13] C. Duhr, S. Hoeche and F. Maltoni, Color-dressed recursive relations for multi-parton amplitudes, JHEP 08 (2006) 062 [hep-ph/0607057] [INSPIRE].

[14] S. Weinzierl, Tales of 1001 Gluons, Phys. Rept. 676 (2017) 1 [arXiv:1610.05318] [INSPIRE].

[15] Z. Bern, J.J.M. Carrasco and H. Johansson, New Relations for Gauge-Theory Amplitudes, Phys. Rev. D 78 (2008) 085011 [arXiv:0805.3993] [InSPIRE].

[16] Z. Bern, J.J.M. Carrasco and H. Johansson, Perturbative Quantum Gravity as a Double Copy of Gauge Theory, Phys. Rev. Lett. 105 (2010) 061602 [arXiv: 1004.0476] [INSPIRE].

[17] Z. Bern, T. Dennen, Y.-t. Huang and M. Kiermaier, Gravity as the Square of Gauge Theory, Phys. Rev. D 82 (2010) 065003 [arXiv: 1004.0693] [INSPIRE].

[18] P. Griffiths and J. Harris, Principles of Algebraic Geometry, John Wiley \& Sons, New York, U.S.A. (1994).

[19] S. Abreu, R. Britto, C. Duhr and E. Gardi, Cuts from residues: the one-loop case, JHEP 06 (2017) 114 [arXiv:1702.03163] [INSPIRE].

[20] P. Deligne and D. Mumford, The irreducibility of the space of curves of given genus, Publ. Math. Inst. Hautes É tudes Sci. 36 (1969) 75.

[21] F. Knudsen and D. Mumford, The projectivity of the moduli space of stable curves I: Preliminaries on "det" and "Div", Math. Scand. 39 (1976) 19.

[22] F. Knudsen, The projectivity of the moduli space of stable curves II: The stacks $M_{g, n}$, Math. Scand. 52 (1983) 161.

[23] F. Knudsen, The projectivity of the moduli space of stable curves III: The line bundles on $M_{g, n}$, and a proof of the projectivity of $\bar{M}_{g, n}$ in characteristic 0, Math. Scand. 52 (1983) 200.

[24] F. Brown, Multiple zeta values and periods of moduli spaces $\overline{\mathcal{M}}_{0, n}$, C. R. Acad. Sci. Paris 342 (2006) 949.

[25] J.D. Stasheff, Homotopy associativity of H-spaces. I, Trans. Amer. Math. Soc. 108 (1963) 275 .

[26] J.D. Stasheff, Homotopy associativity of $H$-spaces. II, Trans. Amer. Math. Soc. 108 (1963) 293.

[27] S. Devadoss, Tessellations of Moduli Spaces and the Mosaic Operad, math/9807010.

[28] S. Devadoss, Combinatorial equivalence of real moduli spaces, Notices of the AMS $\mathbf{5 1}$ (2004) 620 math.ph/0405011. 
[29] R. Kleiss and H. Kuijf, Multi - Gluon Cross-sections and Five Jet Production at Hadron Colliders, Nucl. Phys. B 312 (1989) 616 [InSPIRE].

[30] N. Arkani-Hamed, Spacetime, QM and Positive Geometry, talk presented at Amplitudes 2017, Edinburgh, U.K., 10-14 July 2017.

[31] Y. Bai, Positive Geometries and Canonical Forms, talk presented at Amplitudes 2017,

[32] Y.-J. Du, B. Feng and C.-H. Fu, The Construction of Dual-trace Factor in Yang-Mills Theory, JHEP 07 (2013) 057 [arXiv: 1304. 2978] [INSPIRE].

[33] S. Litsey and J. Stankowicz, Kinematic numerators and a double-copy formula for $N=4$ super-Yang-Mills residues, Phys. Rev. D 90 (2014) 025013 [arXiv:1309.7681] [INSPIRE].

[34] C.S. Lam and Y.-P. Yao, Evaluation of the Cachazo-He-Yuan gauge amplitude, Phys. Rev. D 93 (2016) 105008 [arXiv:1602.06419] [INSPIRE].

[35] N.E.J. Bjerrum-Bohr, J.L. Bourjaily, P.H. Damgaard and B. Feng, Manifesting Color-Kinematics Duality in the Scattering Equation Formalism, JHEP 09 (2016) 094 [arXiv: 1608.00006] [INSPIRE].

[36] R. Huang, Y.-J. Du and B. Feng, Understanding the Cancelation of Double Poles in the Pfaffian of CHY-formulism, JHEP 06 (2017) 133 [arXiv:1702.05840] [INSPIRE].

[37] Y.-J. Du and F. Teng, BCJ numerators from reduced Pfaffian, JHEP 04 (2017) 033 [arXiv: 1703.05717] [INSPIRE].

[38] X. Gao, S. He and Y. Zhang, Labelled tree graphs, Feynman diagrams and disk integrals, JHEP 11 (2017) 144 [arXiv:1708.08701] [INSPIRE].

[39] T. Wang, G. Chen, Y.-K.E. Cheung and F. Xu, A differential operator for integrating one-loop scattering equations, JHEP 01 (2017) 028 [arXiv: 1609.07621] [INSPIRE].

[40] T. Wang, G. Chen, Y.-K.E. Cheung and F. Xu, A Combinatoric Shortcut to Evaluate CHY-forms, JHEP 06 (2017) 015 [arXiv:1701.06488] [INSPIRE].

[41] G. Chen and T. Wang, BCJ Numerators from Differential Operator of Multidimensional Residue, arXiv: 1709.08503 [INSPIRE].

[42] E.K. Leinartas, Factorization of rational functions of several variables into partial fractions, Izv. Vyssh. Uchebn. Zaved. Mat. 22 (1978) 47.

[43] A. Raichev, Leinartas's partial fraction decomposition, arXiv:1206.4740.

[44] C. Meyer, Transforming differential equations of multi-loop Feynman integrals into canonical form, JHEP 04 (2017) 006 [arXiv: 1611.01087] [INSPIRE].

[45] N. Arkani-Hamed and J. Trnka, The Amplituhedron, JHEP 10 (2014) 030 [arXiv: 1312.2007] [INSPIRE].

[46] N. Arkani-Hamed and J. Trnka, Into the Amplituhedron, JHEP 12 (2014) 182 [arXiv: 1312.7878] [INSPIRE].

[47] N. Arkani-Hamed, A. Hodges and J. Trnka, Positive Amplitudes In The Amplituhedron, JHEP 08 (2015) 030 [arXiv: 1412.8478] [INSPIRE].

[48] Y. Bai and S. He, The Amplituhedron from Momentum Twistor Diagrams, JHEP 02 (2015) 065 [arXiv: 1408.2459] [INSPIRE].

[49] S. Franco, D. Galloni, A. Mariotti and J. Trnka, Anatomy of the Amplituhedron, JHEP 03 (2015) 128 [arXiv:1408.3410] [InSPIRE]. 
[50] Z. Bern, E. Herrmann, S. Litsey, J. Stankowicz and J. Trnka, Evidence for a Nonplanar Amplituhedron, JHEP 06 (2016) 098 [arXiv: 1512.08591] [INSPIRE].

[51] F. Cachazo, S. He and E.Y. Yuan, Scattering Equations and Matrices: From Einstein To Yang-Mills, DBI and NLSM, JHEP 07 (2015) 149 [arXiv:1412.3479] [INSPIRE].

[52] L. de la Cruz, A. Kniss and S. Weinzierl, Proof of the fundamental BCJ relations for QCD amplitudes, JHEP 09 (2015) 197 [arXiv: 1508.01432] [INSPIRE].

[53] L. de la Cruz, A. Kniss and S. Weinzierl, The CHY representation of tree-level primitive QCD amplitudes, JHEP 11 (2015) 217 [arXiv:1508.06557] [INSPIRE].

[54] L. de la Cruz, A. Kniss and S. Weinzierl, Double Copies of Fermions as Matter that Interacts Only Gravitationally, Phys. Rev. Lett. 116 (2016) 201601 [arXiv: 1601.04523] [INSPIRE].

[55] F. Cachazo, S. He and E.Y. Yuan, Einstein- Yang-Mills Scattering Amplitudes From Scattering Equations, JHEP 01 (2015) 121 [arXiv:1409.8256] [INSPIRE].

[56] S. Stieberger and T.R. Taylor, New relations for Einstein-Yang-Mills amplitudes, Nucl. Phys. B 913 (2016) 151 [arXiv: 1606.09616] [INSPIRE].

[57] D. Nandan, J. Plefka, O. Schlotterer and C. Wen, Einstein- Yang-Mills from pure Yang-Mills amplitudes, JHEP 10 (2016) 070 [arXiv:1607.05701] [INSPIRE].

[58] L. de la Cruz, A. Kniss and S. Weinzierl, Relations for Einstein-Yang-Mills amplitudes from the CHY representation, Phys. Lett. B 767 (2017) 86 [arXiv: 1607.06036] [InSPIRE].

[59] C.-H. Fu, Y.-J. Du, R. Huang and B. Feng, Expansion of Einstein- Yang-Mills Amplitude, JHEP 09 (2017) 021 [arXiv:1702.08158] [INSPIRE].

[60] F. Teng and B. Feng, Expanding Einstein-Yang-Mills by Yang-Mills in CHY frame, JHEP 05 (2017) 075 [arXiv: 1703.01269] [INSPIRE].

[61] M. Chiodaroli, M. Günaydin, H. Johansson and R. Roiban, Explicit Formulae for Yang-Mills-Einstein Amplitudes from the Double Copy, JHEP 07 (2017) 002 [arXiv: 1703.00421] [INSPIRE].

[62] H. Johansson and A. Ochirov, Pure Gravities via Color-Kinematics Duality for Fundamental Matter, JHEP 11 (2015) 046 [arXiv: 1407.4772] [INSPIRE].

[63] H. Johansson and A. Ochirov, Color-Kinematics Duality for QCD Amplitudes, JHEP 01 (2016) 170 [arXiv:1507.00332] [INSPIRE]. 